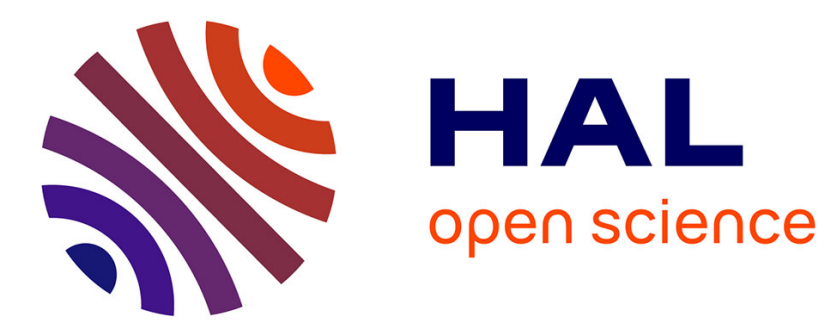

\title{
Generalized stochastic approach for constitutive equation in linear elasticity: a random matrix model
}

Johann Guilleminot, Christian Soize

\section{To cite this version:}

Johann Guilleminot, Christian Soize. Generalized stochastic approach for constitutive equation in linear elasticity: a random matrix model. International Journal for Numerical Methods in Engineering, 2012, 90 (5), pp.613-635. 10.1002/nme.3338 . hal-00699345

\section{HAL Id: hal-00699345 \\ https://hal.science/hal-00699345}

Submitted on 20 May 2012

HAL is a multi-disciplinary open access archive for the deposit and dissemination of scientific research documents, whether they are published or not. The documents may come from teaching and research institutions in France or abroad, or from public or private research centers.
L'archive ouverte pluridisciplinaire HAL, est destinée au dépôt et à la diffusion de documents scientifiques de niveau recherche, publiés ou non, émanant des établissements d'enseignement et de recherche français ou étrangers, des laboratoires publics ou privés. 


\title{
Generalized Stochastic Approach for Constitutive Equation in Linear Elasticity: A Random Matrix Model*
}

\author{
J. Guilleminot, C. Soize \\ Université Paris-Est \\ Laboratoire MSME UMR8208 CNRS \\ 5 Bd Descartes, 77454 Marne la Vallée, France
}

12 DEC 2011

\begin{abstract}
This work is concerned with the construction of stochastic models for random elasticity matrices, allowing either for the generation of elasticity tensors exhibiting some material symmetry properties almost surely (integrating the statistical dependence between the random stiffness components), or for the modeling of random media that requires the mean of a stochastic anisotropy measure to be controlled apart from the level of statistical fluctuations. To this aim, we first introduce a decomposition of the stochastic elasticity tensor on a deterministic tensor basis and consider the probabilistic modeling of the random components, having recourse to the MaxEnt principle. Strategies for random generation and estimation are further reviewed and the approach is exemplified in the case of a material that is transversely isotropic almost surely. In a second stage, we make use of such derivations to propose a generalized model for random elasticity matrices that takes into account, almost separately, constraints on both the level of stochastic anisotropy and the level of statistical fluctuations. An example is finally provided and shows the efficiency of the approach.
\end{abstract}

Keywords: Elasticity tensors; Probabilistic model; Material symmetry; Random matrix.

\section{INTRODUCTION}

This work is concerned with the modeling of the stochastic constitutive law in linear elasticity and more precisely, with the probabilistic modeling of random elasticity tensors. Such an issue arises in many situations and has been addressed in various fields of applications over the past twenty years.

From a practical point of view, mechanical engineers are generally interested in getting stochastic models that can be readily implemented in either commercial or in-house codes, while being both mathematically sound and physically consistent. For the sake of theoretical or numerical convenience, (industrial) applications are performed, in most cases, assuming that the materials, while exhibiting a random behavior, do preserve some symmetry properties. In this context, pieces of steel (resp. composite unidirectional plies made up with either isotropic or transversely isotropic fibers) are usually considered as being isotropic

* J. Guilleminot and C. Soize, Generalized Stochastic Approach for Constitutive Equation in Linear Elasticity: A Random Matrix Model, International Journal for Numerical Methods in Engineering, available online, DOI: 10.1002/nme.3338 (2011). 
(resp. transversely isotropic), for instance. A typical question that one may then face is the dependence between the components of the elasticity tensor: are these quantities statistically dependent? If so, how should such a dependence be taken into account and would it remain the same if the parametrization is changed (e.g. considering the so-called engineering constants)? Clearly, physics suggests that the answer to the first question may be affirmative (but does not tell how and to what extent though), hence emphasizing the need for developing stochastic models able to generate realizations belonging to a set of tensors with given symmetries and allowing for any potential statistical dependence to be integrated.

From a more theoretical standpoint, a lot of attention has been paid on probabilistic modeling in elastostatics, mainly within the scope of mesoscale modeling for random, heterogeneous media. Although the first fundamental works on the so-called apparent (random) elasticity tensor date back to the early nineties [17] [34] (see the review in [32]), the field has become very popular quite recently, benefiting from the tremendous developments around the Stochastic Finite Elements Method (SFEM; see the monograph by Ghanem and Spanos [9]), and has served as a basis for many studies related to stochastic solvers for reliability analysis, stochastic inverse identification, optimal (robust) design, etc. In this general framework of mesoscale modeling, two fundamental types of approach can be basically distinguished, differing essentially in the nature of the (experimental) data that are assumed to be available. When digital microstructures of the material can be obtained, mathematical-morphology-based approaches [35] [46], initially proposed for computational homogenization purposes, have been used to generate and/or calibrate random fields of local properties which can be used afterwards as a physically and micromechanics sound basis for SFEM applications. Such an approach has been pioneered in [31] in linear elasticity and was recently revisited, using more advanced upscaling/representation techniques and for nonlinear behavior, in [10] (see also [45] [1] [49], among others). When such data are not available, one is typically concerned with the construction of (prior) algebraic stochastic representations that can be used for identifying a random field of properties by solving an inverse problem associated with a stochastic boundary value problem; see [40] and [13] for such constructions, and [43] for a discussion regarding the identification methodology. Restricting ourselves to the case of linear elasticity, it is well-known that the realizations of the mesoscopic elasticity tensor do not perfectly exhibit given material symmetries. Indeed, some more or less anisotropic fluctuations around a symmetry class are often observed, the latter being usually the one expected at the macroscale. Consequently, there is arguably a need for stochastic models which allows one to control some (statistical) properties related to any stochastic anisotropy measure, together with and apart from the level of statistical fluctuations. Such an issue has been addressed very differently in [44] and [12], although the two approaches are both derived by invoking the Maximum Entropy principle (see Section 3.1.2). In the first case, the information regarding the symmetry constraint has been integrated by using a particular algebraic representation for the random elasticity tensor, while the second methodology relies on an eigensystem-based characterization of the material symmetry classes (see [3]). However, the approach developed in [44] is restricted to the case of almost isotropic materials and the freedom of prescribing the mean of the anisotropy measure turns out to be somewhat limited (because of the repulsion phenomena of the random eigenvalues) in some cases for the second approach.

In this work, we address the aforementioned issues from a random matrix point of view, that are:

(i) the construction of a stochastic model which allows for the generation of elasticity matrices exhibiting some material symmetry properties almost surely, integrating some statistical dependence between the random stiffness components;

(ii) the derivation of a probabilistic representation that allows one to model the apparent elasticity tensor associated with any random media at the mesoscale, taking into account the prescription of 
some statistical features of a stochastic anisotropy measure and the level of fluctuations apart from one another, and that for all linear elasticity symmetries.

This paper is organized as follows. We first discuss, in Section 2, the definition of an overall decomposition which aims at ensuring an uniform ellipticity condition for the elasticity random bilinear form. In Section 3 , we address the construction of a probabilistic model for elasticity random matrices that exhibit, almost surely, some symmetry properties. For this purpose, we introduce a decomposition of the stochastic elasticity tensor on a deterministic tensor basis and consider the probabilistic modeling of the random components. Strategies for random generation and estimation are further reviewed and the approach is exemplified in the case of a material that is transversely isotropic almost surely. In Section 4, we make use of such derivations to propose a generalized model for random elasticity matrices that takes into account, by introducing an additional source of random anisotropy, constraints on both the level of stochastic anisotropy and the level of statistical fluctuations. An example is finally provided and shows the efficiency of the approach.

Notations. Throughout the paper, abbreviation 'a.s.' stands either for 'almost surely' or 'almost sure'.

For any vectors $\boldsymbol{x}=\left(x_{1}, \ldots, x_{n}\right)$ and $\boldsymbol{y}=\left(y_{1}, \ldots, y_{n}\right)$ in $\mathbb{R}^{n}$, we denote as $\langle\boldsymbol{x}, \boldsymbol{y}\rangle=\sum_{i=1}^{n} x_{i} y_{i}$ the Euclidean inner product in $\mathbb{R}^{n}$ and as $\|\cdot\|$ the associated norm, $\left.\|\boldsymbol{x}\|^{2}=<\boldsymbol{x}, \boldsymbol{x}\right\rangle$.

Let $\mathbb{M}_{n}^{S}(\mathbb{R})$ and $\mathbb{M}_{n}^{+}(\mathbb{R})$ be the sets of all the $(n \times n)$ real symmetric matrices and the $(n \times n)$ real symmetric positive-definite matrices $\left(\mathbb{M}_{n}^{+}(\mathbb{R}) \subset \mathbb{M}_{n}^{S}(\mathbb{R})\right)$, respectively. The determinant, trace and transpose of matrix $[A] \in \mathbb{M}_{n}(\mathbb{R})\left(\mathbb{M}_{n}(\mathbb{R})\right.$ being the set of all the square $(n \times n)$ real matrices $)$ are denoted by $\operatorname{det}([A]), \operatorname{tr}([A])$ and $[A]^{\mathrm{T}}$, respectively. For any random matrix $[\boldsymbol{A}]$ with values in $\mathbb{M}_{n}^{+}(\mathbb{R})$, we denote by $\delta_{[\boldsymbol{A}]}$ the parameter measuring the level of fluctuations of $[\boldsymbol{A}]$, defined as:

$$
\delta_{[\boldsymbol{A}]}^{2}=\mathrm{E}\left\{\|[\boldsymbol{A}]-[\underline{A}]\|_{\mathrm{F}}^{2}\right\} /\|[\underline{A}]\|_{\mathrm{F}}^{2},
$$

where $\|[A]\|_{\mathrm{F}}^{2}=\operatorname{tr}\left([A]^{2}\right)$ for $[A]$ in $\mathbb{M}_{n}(\mathbb{R})$ and $[\underline{A}]=\mathrm{E}\{[\boldsymbol{A}]\}$. All inequalities between positive-definite matrices are defined with respect to the usual positive definite (Loewner) ordering (see Section 7.7, pp. 469 of [16], for instance).

\section{PRELIMINARY DECOMPOSITION OF THE RANDOM ELAS- TICITY TENSOR}

Let $\mathbb{E}$ la be the set of fourth-order elasticity tensors. In this paper, we use double brackets (e.g. $[[C]]$, $[[\boldsymbol{C}]]$, etc.) and single brackets (e.g. $[C],[\boldsymbol{C}]$, etc.) to denote $\mathbb{E}$ la-valued fourth-order elasticity tensors and $\mathbb{M}_{n}^{+}(\mathbb{R})$-valued elasticity matrix representations (using the so-called Kelvin's form; see [25] for a discussion), regardless of their deterministic or random nature.

Let $[\widetilde{\boldsymbol{C}}]$ be the random elasticity matrix for which the probabilistic model has to be derived. Let us assume that the mean value $[\underline{\widetilde{C}}]=\mathrm{E}\{[\widetilde{\boldsymbol{C}}]\}$ is known, and write $[\widetilde{\boldsymbol{C}}]$ as

$$
[\widetilde{\boldsymbol{C}}]=\left[C_{\ell}\right]+[\boldsymbol{C}],
$$

in which $\left[C_{\ell}\right] \in \mathbb{M}_{n}^{+}(\mathbb{R})$ is a deterministic matrix and $[\boldsymbol{C}]$ is a $\mathbb{M}_{n}^{+}(\mathbb{R})$-valued random variable, such that:

$$
\mathrm{E}\{[\boldsymbol{C}]\}=[\underline{C}]=[\underline{\widetilde{C}}]-\left[C_{\ell}\right]>0 .
$$


Consequently, since $[\boldsymbol{C}]=[\widetilde{\boldsymbol{C}}]-\left[C_{\ell}\right]>0$, it can be deduced that $[\widetilde{\boldsymbol{C}}]$ is almost surely bounded from below by $\left[C_{\ell}\right]$ :

$$
[\widetilde{\boldsymbol{C}}]>\left[C_{\ell}\right] .
$$

Such a lower bound can be defined in two ways:

(i) If some microstructural information is available, $\left[C_{\ell}\right]$ may be computed, either by using some wellknown micromechanics-based bounds (such as the Reuss bound, for heterogeneous materials made up with ordered phases with deterministic properties) or by using a numerical approximation based on the realizations of the stochastic lower bound obtained from computational homogenization and invoking Huet's Partition Theorem [17]; see the discussion in [11].

(ii) In the absence of such information, a simple a priori expression for $\left[C_{\ell}\right]$ can be obtained as

$$
\left[C_{\ell}\right]=\epsilon[\widetilde{\widetilde{C}}]
$$

with $0 \leq \epsilon<1$, from which it can be deduced that $[\underline{C}]=(1-\epsilon)[\underline{\widetilde{C}}]$.

Note that Eq. (2) has been basically introduced in order an uniform ellipticity condition (for the elasticity stochastic bilinear form) to be recovered and further ensures the mechanical consistency when the bound is determined from a micromechanical analysis (see the point (i) above). In practice, the probabilistic models can then be constructed for random variable $[\boldsymbol{C}]$, and the stochastic representations that will derived in Sections 3 and 4 have to be understood as stochastic models for random variable $[\boldsymbol{C}]$ as defined in Eq. (2).

\section{STOCHASTIC MODELING FOR RANDOM MATRICES WITH VALUES IN A GIVEN ARBITRARY MATERIAL SYMME- TRY CLASS}

\subsection{MaxEnt-based derivation of the probabilistic model}

\subsubsection{Algebraic stochastic representation.}

Let $\mathbb{E l a}{ }^{\text {sym }} \subseteq \mathbb{E}$ la be the set of fourth-order elasticity tensors belonging to the material symmetry class 'sym'. We denote by $\left[\left[\boldsymbol{C}^{\mathrm{sym}}\right]\right]$ the $\mathbb{E} l a^{\text {sym }}$-valued random elasticity tensor, which can be decomposed as

$$
\left[\left[\boldsymbol{C}^{\mathrm{sym}}\right]\right]=\sum_{i=1}^{N} C_{i}\left[\left[E_{\mathrm{sym}}{ }^{(i)}\right]\right],
$$

where $\left\{\left[\left[E_{\mathrm{sym}}{ }^{(i)}\right]\right]\right\}_{i=1}^{N}$ is a deterministic tensor basis of $\mathbb{E l a}{ }^{\mathrm{sym}}$ and $\left\{C_{i}\right\}_{i=1}^{N}$ is a set of random coefficients, the probability distributions of which must be determined and which satisfy a.s. some algebraic constraints related to the (a.s.) positive-definiteness of $\left[\left[\boldsymbol{C}^{\mathrm{sym}}\right]\right]$.

The value of parameter $N$ is recalled in Table 1 for the eight linear elastic symmetries, the latter being defined with respect to the usual classification; see [6] and the references therein. The definition of tensor basis for various classes of symmetry has been investigated by numerous authors, among which Walpole (see Section II.B of Reference [47] for instance) and Kunin [24] to name a few. Hereafter, we make use of 


\begin{tabular}{|c|c|}
\hline Material symmetry & $N$ \\
\hline Isotropic & 2 \\
Cubic & 3 \\
Transversely isotropic & 5 \\
Trigonal & 6 \\
Tetragonal & 6 or 7 \\
Orthotropic & 9 \\
Monoclinic & 13 \\
Triclinic & 21 \\
\hline
\end{tabular}

Table 1: Dimension $N$ for all material symmetry classes.

Walpole's formalism and specifically, we follow the notations and framework proposed in [48]. Therefore, the stochastic elasticity matrix $\left[\boldsymbol{C}^{\mathrm{sym}}\right]$ is now written as

$$
\left[\boldsymbol{C}^{\mathrm{sym}}\right]=\sum_{i=1}^{N} C_{i}\left[E_{\mathrm{sym}}{ }^{(i)}\right]
$$

wherein $\left[E_{\mathrm{sym}}{ }^{(i)}\right], i=1, \ldots, N$, is the deterministic matrix representation of fourth-order basis tensor $\left[\left[E_{\mathrm{sym}}{ }^{(i)}\right]\right]$. Hence, the construction of a probabilistic model for random elasticity tensor $\left[\left[\boldsymbol{C}^{\text {sym }}\right]\right]$ is strictly equivalent to the construction of a model for random coordinates $C_{1}, \ldots, C_{N}$, and such an issue is now to be addressed in Section 3.1.2.

\subsubsection{Model derivation.}

Let $\boldsymbol{C}=\left(C_{1}, \ldots, C_{N}\right)$ be the $\mathbb{R}^{N}$-valued second-order random variable, corresponding to the modeling of the random coordinates of $\left[\boldsymbol{C}^{\text {sym }}\right]$ onto $\left\{\left[E_{\mathrm{sym}}{ }^{(i)}\right]\right\}_{i=1}^{N}$ and whose unknown probability distribution $P_{\boldsymbol{C}}(\mathrm{d} \boldsymbol{c})$ is defined by a probability density function (p.d.f.) $p_{\boldsymbol{C}}$ with respect to the Lebesgue measure $\mathrm{d} \boldsymbol{c}=\mathrm{d} c_{1} \ldots \mathrm{d} c_{N}$. We denote by $\mathcal{S}$ the support of $p_{\boldsymbol{C}}$. Because of the a.s. positive-definiteness of $\left[\boldsymbol{C}^{\mathrm{sym}}\right]$, it follows that $\mathcal{S}$ is a part, possibly unbounded, of $\mathbb{R}^{N}$, the definition of which depends on the considered material symmetry class. For instance, one has $\mathcal{S}=\mathbb{R}^{+} \times \mathbb{R}^{+}$for the isotropic case, and the case of materials which are transversely isotropic a.s. will be investigated later in Section 3.3.

The probabilistic model for random vector $\boldsymbol{C}$ is constructed by having recourse to the Maximum Entropy (MaxEnt) principle, which is a general optimization procedure, derived within the framework of information theory [36], which allows for the explicit construction of probability distributions under a set of constraints defining some available information [18]; see also [7] [19]. Specifically, let us consider the two constraints

$$
\mathrm{E}\{\boldsymbol{C}\}=\underline{\boldsymbol{c}}, \underline{\boldsymbol{c}}=\left(\underline{c}_{1}, \ldots, \underline{c}_{N}\right)
$$

and

$$
\mathrm{E}\left\{\log \left(\operatorname{det}\left(\sum_{i=1}^{N} C_{i}\left[E_{\mathrm{sym}}{ }^{(i)}\right]\right)\right)\right\}=\nu_{\boldsymbol{C}},\left|\nu_{\boldsymbol{C}}\right|<+\infty,
$$

together with the usual normalization condition

$$
\int_{\mathcal{S}} p_{\boldsymbol{C}}(\boldsymbol{c}) \mathrm{d} \boldsymbol{c}=1 .
$$


Eq. (8) means that the mean value of $\boldsymbol{C}$ is supposed to be known and parameter $\underline{\boldsymbol{c}}$ could correspond, in practice, to the nominal value of the coordinates, should a deterministic framework be adopted. The constraint given by Eq. (9) (i) allows one to prove that both $\left[\boldsymbol{C}^{\text {sym }}\right]$ and $\left[\boldsymbol{C}^{\text {sym }}\right]^{-1}$ are second-order random variables (see [37] [38]) and (ii) potentially creates a statistical dependence between the components of random vector $\boldsymbol{C}$ (see the application below).

Let $\boldsymbol{\lambda}^{(1)} \in \mathcal{A}_{\boldsymbol{\lambda}^{(1)}}, \lambda^{(2)} \in \mathcal{A}_{\lambda^{(2)}}$ and $\lambda^{(0)} \in \mathbb{R}^{+}$be the Lagrange multipliers associated with constraints (8), (9) and (10) respectively. Let $\boldsymbol{\lambda}=\left(\boldsymbol{\lambda}^{(1)}, \lambda^{(2)}\right) \in \mathcal{A}_{\boldsymbol{\lambda}}$, with $\mathcal{A}_{\boldsymbol{\lambda}}=\mathcal{A}_{\boldsymbol{\lambda}^{(1)}} \times \mathcal{A}_{\boldsymbol{\lambda}^{(2)}}$, and let $\lambda_{\text {sol }}^{(0)}$ and $\boldsymbol{\lambda}_{\text {sol }}=\left(\boldsymbol{\lambda}_{\text {sol }}^{(1)}, \lambda_{\text {sol }}^{(2)}\right)$ be the solution Lagrange multipliers such that Eqs. (8), (9) and (10) are satisfied. It can then be shown that the p.d.f. $p_{\boldsymbol{C}}$ takes the form

$$
p_{\boldsymbol{C}}(\boldsymbol{c})=\mathbb{1}_{\mathcal{S}}(\boldsymbol{c}) k_{\mathrm{sol}} \exp \left\{-<\boldsymbol{\lambda}_{\mathrm{sol}}, \boldsymbol{g}(\boldsymbol{c})>_{\mathbb{R}^{N+1}}\right\},
$$

in which $\boldsymbol{c} \mapsto \mathbb{1}_{\mathcal{S}}(\boldsymbol{c})$ is the indicator (characteristic) function of $\mathcal{S}, k_{\text {sol }}=\exp \left\{-\lambda_{\text {sol }}^{(0)}\right\}$ is the normalizing constant (which basically depends on $\boldsymbol{\lambda}_{\text {sol }}$ ), $\boldsymbol{a} \mapsto \boldsymbol{g}(\boldsymbol{a})$ is the mapping defined on $\mathcal{S} \times \mathbb{R}$, with values in $\mathbb{R}^{N+1}$, such that $\boldsymbol{g}(\boldsymbol{a})=(\boldsymbol{a}, \varphi(\boldsymbol{a}))$. The mapping $\varphi: \mathcal{S} \longrightarrow \mathbb{R}$ is defined as:

$$
\varphi(\boldsymbol{c})=\log \left(\operatorname{det}\left(\sum_{i=1}^{N} c_{i}\left[E_{\mathrm{sym}}{ }^{(i)}\right]\right)\right) .
$$

It is worth while to note at this stage that the admissible space $\mathcal{A}_{\boldsymbol{\lambda}}$ for the Lagrange multipliers must be defined in order to ensure the integrability of the p.d.f. (11) at both the origin and infinity (see Section 3.3.2 for an illustration).

In view of the multipliers identification, let us introduce, for $\boldsymbol{\lambda}$ fixed in $\mathcal{A}_{\boldsymbol{\lambda}}$, the $\mathbb{R}^{N}$-valued random variable $\boldsymbol{D}_{\boldsymbol{\lambda}}$, the probability density function of which is denoted by $p_{\boldsymbol{D}_{\boldsymbol{\lambda}}}$, has support $\mathcal{S} \subset \mathbb{R}^{N}$ and is written as

$$
p_{\boldsymbol{D}_{\boldsymbol{\lambda}}}(\boldsymbol{d} ; \boldsymbol{\lambda})=\mathbb{1}_{\mathcal{S}}(\boldsymbol{d}) k_{\boldsymbol{D}_{\boldsymbol{\lambda}}} \exp \left\{-<\boldsymbol{\lambda}, \boldsymbol{g}(\boldsymbol{d})>_{\mathbb{R}^{N+1}}\right\},
$$

where $k_{\boldsymbol{D}_{\boldsymbol{\lambda}}}=k_{\boldsymbol{D}_{\boldsymbol{\lambda}}}(\boldsymbol{\lambda})$ is the positive constant of normalization. Thus, it can be deduced that

$$
C=D_{\lambda_{\text {sol }}}
$$

for the convergence in probability distribution. From Eqs. (8), (9) and the definition of mapping $\boldsymbol{g}$, it turns out that the computation of the Lagrange multiplier $\boldsymbol{\lambda}_{\text {sol }}$ involves the numerical estimation of the mathematical expectation $\mathrm{E}\left\{\boldsymbol{g}\left(\boldsymbol{D}_{\boldsymbol{\lambda}}\right)\right\}$. For this purpose, it is convenient to finally introduce the $\mathbb{R}^{N}$-valued random variable $\boldsymbol{B}_{\boldsymbol{\lambda}}$ whose probability distribution $p_{\boldsymbol{B}_{\boldsymbol{\lambda}}}$, defined from $\mathbb{R}^{N}$ into $\mathbb{R}^{+}$, is given by

$$
p_{\boldsymbol{B}_{\boldsymbol{\lambda}}}(\boldsymbol{b} ; \boldsymbol{\lambda})=k_{\boldsymbol{B}_{\boldsymbol{\lambda}}} \exp \left\{-<\boldsymbol{\lambda}, \boldsymbol{g}(\boldsymbol{b})>_{\mathbb{R}^{N+1}}\right\}
$$

with $k_{\boldsymbol{B}_{\boldsymbol{\lambda}}}$ the normalization constant. For $\boldsymbol{\lambda}$ fixed in $\mathcal{A}_{\boldsymbol{\lambda}}$, it can be easily shown that $\mathrm{E}\left\{\boldsymbol{g}\left(\boldsymbol{D}_{\boldsymbol{\lambda}}\right)\right\}$ writes [41]

$$
\mathrm{E}\left\{\boldsymbol{g}\left(\boldsymbol{D}_{\boldsymbol{\lambda}}\right)\right\}=\frac{\mathrm{E}\left\{\mathbb{1}_{\mathcal{S}}\left(\boldsymbol{B}_{\boldsymbol{\lambda}}\right) \boldsymbol{g}\left(\boldsymbol{B}_{\boldsymbol{\lambda}}\right)\right\}}{\mathrm{E}\left\{\mathbb{1}_{\mathcal{S}}\left(\boldsymbol{B}_{\boldsymbol{\lambda}}\right)\right\}} .
$$

Consequently, solving for parameter $\boldsymbol{\lambda}$ can be performed by first generating independent realizations of the random variable $\boldsymbol{B}_{\boldsymbol{\lambda}}$ whose p.d.f. is supported over $\mathbb{R}^{N}$, and then by estimating the r.h.s. of Eq. (15) using any appropriate (e.g. Monte Carlo) method (see the discussion below). Such numerical issues, together with the computation of the Lagrange multipliers, are addressed in the next section. 


\subsection{Computational aspects}

\subsubsection{Random generator for random variable $B_{\lambda}$.}


dent realizations of $\boldsymbol{B}_{\boldsymbol{\lambda}}$ with respect to p.d.f. (14). The Markov Chain Monte Carlo (MCMC) method [33] is a natural choice to this aim and one may have recourse to either the Metropolis-Hastings (MH) [27] [26] [15], Gibbs [8] or Slice Sampling (SS) [30] algorithm. Nevertheless, the first (MH) technique is tunable, for it requires the definition of a (good) proposal distribution, and the second one requires the knowledge of the conditional distributions. On the contrary, the SS technique requires neither the choice of a proposal distribution nor the calculation of the conditional distributions, but may not perform well depending on the general shape of the joint p.d.f. (and especially, in the case of multi-modal distributions). Consequently, such algorithms, while appealing from a theoretical standpoint, could be tricky to use in practice as the dimension $N$ becomes quite large (see Table 1), and automatic robust calculations (which are desired within the main optimization loop for the computation of the optimal Lagrange multiplier $\boldsymbol{\lambda}_{\text {sol }}$, regardless of the current value of the guess at a given iteration) may not be obtainable. It should be pointed out that the definition of an adaptive MCMC technique could be investigated, in which some given parameters (e.g. some parameters of the proposal distribution for the MH sampler) would be tailored while running the chain. Such a path would, however, give rise to mathematical concerns (since the chain may not be Markovian anymore, etc.) which are beyond the scope of the proposed work. In this paper, we subsequently make use of an alternative algorithm, introduced by Soize in [41], the construction of which similarly involves the construction of an ergodic homogeneous Markov chain and which is briefly summarized below for the sake of self-readability. As will be seen, such a technique lies upon a firm and well-defined mathematical footing, benefits from its robustness and unlike the few other MCMC algorithms mentioned above, does have a parametrization that is completely independent from the dimension parameter $N$.

Let us first introduce $\boldsymbol{u} \mapsto \Phi\left(\boldsymbol{u} ; \boldsymbol{\lambda}^{(1)}, \lambda^{(2)}\right)$ be the so-called potential function $\Phi$ defined as:

$$
\Phi(\boldsymbol{u} ; \boldsymbol{\lambda})=<\boldsymbol{\lambda}, \boldsymbol{g}(\boldsymbol{u})>_{\mathbb{R}^{N+1}} .
$$

Let $\boldsymbol{W}$ be the normalized Wiener process defined on $(\Theta, \mathcal{T}, \mathcal{P})$, indexed by $\mathbb{R}^{+}$and with values in $\mathbb{R}^{N}$. Let $(\boldsymbol{U}, \boldsymbol{V})$ be the Markov stochastic process, defined on probability space $(\Theta, \mathcal{T}, \mathcal{P})$, indexed by $\mathbb{R}^{+}$, with values in $\mathbb{R}^{N} \times \mathbb{R}^{N}$ and satisfying the following Itô stochastic differential equation (ISDE)

$$
\forall r \in \mathbb{R}^{+},\left\{\begin{array}{l}
\mathrm{d} \boldsymbol{U}(r)=\boldsymbol{V}(r) \mathrm{d} r \\
\mathrm{~d} \boldsymbol{V}(r)=-\nabla_{\boldsymbol{u}} \Phi(\boldsymbol{U}(r) ; \boldsymbol{\lambda}) \mathrm{d} r-\frac{f_{0}}{2} \boldsymbol{V}(r) \mathrm{d} r+\sqrt{f_{0}} \mathrm{~d} \boldsymbol{W}(r)
\end{array},\right.
$$

under given initial conditions $\boldsymbol{U}(0)=\boldsymbol{U}_{0}$ and $\boldsymbol{V}(0)=\boldsymbol{V}_{0}$ a.s., where the probability distribution of $\left(\boldsymbol{U}_{0}, \boldsymbol{V}_{0}\right)$ is assumed to be known. The parameter $f_{0}$ is a positive real parameter which must be selected in order to obtain a reasonably fast convergence to the stationary solution of the ISDE. Under some given assumptions (see Eqs. (23), (24) and (25) of Reference [41]) that are assumed to be satisfied, it can be shown that

$$
\lim _{r \rightarrow+\infty} \boldsymbol{U}(r)=\boldsymbol{B}_{\boldsymbol{\lambda}}
$$

in probability distribution [41]. Consequently, it follows that independent realizations $\boldsymbol{B}_{\boldsymbol{\lambda}}\left(\theta_{1}\right), \ldots, \boldsymbol{B}_{\boldsymbol{\lambda}}\left(\theta_{n_{s}}\right)$ of random variable $\boldsymbol{B}_{\boldsymbol{\lambda}}$ can be obtained as

$$
\boldsymbol{B}_{\boldsymbol{\lambda}}\left(\theta_{\ell}\right)=\boldsymbol{U}\left(r, \theta_{\ell}\right), \ell=1, \ldots, n_{s},
$$


for $r$ sufficiently large, where $\left(\boldsymbol{U}\left(r, \theta_{\ell}\right), \boldsymbol{V}\left(r, \theta_{\ell}\right)\right), r \geq 0$, is the solution of

$$
\forall r \in \mathbb{R}^{+},\left\{\begin{array}{l}
\mathrm{d} \boldsymbol{U}\left(r, \theta_{\ell}\right)=\boldsymbol{V}\left(r, \theta_{\ell}\right) \mathrm{d} r \\
\mathrm{~d} \boldsymbol{V}\left(r, \theta_{\ell}\right)=-\nabla_{\boldsymbol{u}} \Phi\left(\boldsymbol{U}\left(r, \theta_{\ell}\right) ; \boldsymbol{\lambda}\right) \mathrm{d} r-\frac{f_{0}}{2} \boldsymbol{V}\left(r, \theta_{\ell}\right) \mathrm{d} r+\sqrt{f_{0}} \mathrm{~d} \boldsymbol{W}\left(r, \theta_{\ell}\right)
\end{array},\right.
$$

with initial conditions $\boldsymbol{U}\left(0, \theta_{\ell}\right)=\boldsymbol{u}_{0}$ and $\boldsymbol{V}\left(0, \theta_{\ell}\right)=\boldsymbol{v}_{0}\left(\boldsymbol{u}_{0}\right.$ and $\boldsymbol{v}_{0}$ being two deterministic given vectors in $\left.\mathbb{R}^{N}\right), \boldsymbol{W}\left(r, \theta_{\ell}\right)$ being an independent realization of stochastic process $\boldsymbol{W}$.

In practice, an approximation of the solution is considered and can be obtained using integration schemes, such as the ones detailed in [41] (see [20] for a review). Therefore, the ISDE is solved over an interval $[0,(M-1) \Delta r]$, where $\Delta r$ is a discretization step (which must be small enough), and the $k$-th sampling point $r_{k}$ is defined as $r_{k}=(k-1) \Delta r$. Denoting as $r_{0}=\left(M_{0}-1\right) \Delta r$ the time step such that the stationary solution is reached for all $r_{k} \geq r_{0}$, it can be deduced that an independent realization $\boldsymbol{B}_{\boldsymbol{\lambda}}\left(\theta_{\ell}\right)$ of $\boldsymbol{B}_{\boldsymbol{\lambda}}$ can be obtained as

$$
\boldsymbol{B}_{\boldsymbol{\lambda}}\left(\theta_{\ell}\right)=\boldsymbol{U}\left(r_{k}, \theta_{\ell}\right), r_{k} \geq r_{0} .
$$

As briefly discussed above, the minimal parametrization of the random generator is note worthy, since the latter only depends on parameter $f_{0}$ and sampling step $\Delta r$.

\subsubsection{Computation of the mathematical expectations.}

Based on the previous random generator, there are two possible strategies for computing the mathematical expectations of the r.h.s. of Eq. (15). The first one can be obtained by invoking the Ergodic theorem [50], and the resulting ergodic estimator reads

$$
\mathrm{E}\left\{\boldsymbol{g}\left(\boldsymbol{D}_{\boldsymbol{\lambda}}\right)\right\} \simeq \sum_{k=M_{0}}^{M} \mathbb{1}_{\mathcal{S}}\left(\boldsymbol{U}\left(r_{k}, \theta_{\ell}\right)\right) \boldsymbol{g}\left(\boldsymbol{U}\left(r_{k}, \theta_{\ell}\right)\right) / \sum_{k=M_{0}}^{M} \mathbb{1}_{\mathcal{S}}\left(\boldsymbol{U}\left(r_{k}, \theta_{\ell}\right)\right),
$$

for a given trajectory $\left\{\boldsymbol{W}\left(r_{k}, \theta_{\ell}\right), k=1, \ldots, M\right\}$ of stochastic process $\boldsymbol{W}$, parameter $M>M_{0}$ being determined from a convergence analysis (see Section 3.3.2 for an application).

Another natural method consists in using independent realizations of random variable $\boldsymbol{B}_{\boldsymbol{\lambda}}$, generated following Eq. (21), and the usual Monte Carlo estimator

$$
\mathrm{E}\left\{\boldsymbol{g}\left(\boldsymbol{D}_{\boldsymbol{\lambda}}\right)\right\} \simeq \sum_{\ell=1}^{n_{s}} \mathbb{1}_{\mathcal{S}}\left(\boldsymbol{U}\left(r_{M}, \theta_{\ell}\right)\right) \boldsymbol{g}\left(\boldsymbol{U}\left(r_{k}, \theta_{\ell}\right)\right) / \sum_{\ell=1}^{n_{s}} \mathbb{1}_{\mathcal{S}}\left(\boldsymbol{U}\left(r_{M}, \theta_{\ell}\right)\right)
$$

where $n_{s}$ must be selected from a convergence analysis and $M>M_{0}$.

From a computational point of view, both methods turn out to be very efficient, the Monte-Carlo method benefiting from a straightforward parallelization. The ergodic estimator requires $M$ iterations within the numerical scheme, while $n_{s} \times M_{0}$ iterations are necessary for obtaining a converged Monte Carlo estimator. In practice, the choice between these methods may then be guided by the values of $M_{0}$, $M$ and $n_{s}$, which must be determined within a preliminary preprocessing step.

\subsubsection{Computation of the Lagrange multipliers.}

Let $\boldsymbol{f}^{\text {target }}=\left(\underline{\boldsymbol{c}}, \nu_{\boldsymbol{C}}\right)$ be the target vector (see Eqs. (8) and (9)), and let $\boldsymbol{f}^{\text {est }}(\boldsymbol{\lambda})=\left(\underline{\widehat{\boldsymbol{c}}}(\boldsymbol{\lambda}), \widehat{\nu}_{\boldsymbol{C}}(\boldsymbol{\lambda})\right.$ ) be the vector of constraints estimated using the random generator (parametrized by $\boldsymbol{\lambda}$ ) and any of the estimation 
methods discussed in Section 3.2.2. The optimal value for the Lagrange multiplier $\boldsymbol{\lambda}$, denoted by $\boldsymbol{\lambda}_{\text {sol }}$, can be computed by solving the following optimization problem

$$
\boldsymbol{\lambda}_{\mathrm{sol}}=\underset{\mathcal{A}_{\boldsymbol{\lambda}}}{\arg \min } \mathcal{J}(\boldsymbol{\lambda})
$$

where the cost function is defined as

$$
\mathcal{J}(\boldsymbol{\lambda})=(1-\alpha)\|\underline{\boldsymbol{c}}-\underline{\widehat{\boldsymbol{c}}}(\boldsymbol{\lambda})\|^{2}+\alpha\left(\nu_{\boldsymbol{C}}-\widehat{\nu}_{\boldsymbol{C}}(\boldsymbol{\lambda})\right)^{2}
$$

and $\alpha \in[0,1]$ is a free parameter. In this study, the constrained optimization problem (24) has been solved using an active-set-type algorithm and the initial guess for the Lagrange multipliers has been determined by making the modes of the distribution match the desired mean values. Since there are $N$ modes and $N+1$ multipliers, the multiplier $\boldsymbol{\lambda}^{(1)}$ related to the mean value constraints is expressed as a function of the target mean values and the remaining multiplier $\lambda^{(2)}$, which is related to the invertibility condition. A parametric study is then performed on the latter alone, and the value that yields a reasonable (i.e. moderate) value for cost function $\mathcal{J}$ is retained for initializing the solving algorithm (see Section 3.3.2).

\subsection{Application to the set of transversely isotropic materials}

In this section, we exemplify the proposed approach by considering the set of transversely isotropic materials, for which $N=5$.

\subsubsection{Tensor decomposition of $[[C]]$ and constraints derivations.}

Let $\mathbb{E} l a^{\text {trans-iso }}$ be the set of fourth-order random elasticity tensors exhibiting transverse isotropy almost surely, and let $\boldsymbol{n}$ be the unit vector normal to the plane of two-dimensional isotropy. The stochastic elasticity tensor $[[\boldsymbol{C}]]$ can then be decomposed as

$$
[[\boldsymbol{C}]]=C_{1}\left[\left[E^{(1)}\right]\right]+C_{2}\left[\left[E^{(2)}\right]\right]+C_{3}\left(\left[\left[E^{(3)}\right]\right]+\left[\left[E^{(4)}\right]\right]\right)+C_{4}[[F]]+C_{5}[[G]],
$$

so that the compact form proposed in [48], allowing for simplified algebraic calculations, reads:

$$
[[\boldsymbol{C}]]=\left\{\left[\begin{array}{ll}
C_{1} & C_{3} \\
C_{3} & C_{2}
\end{array}\right], C_{4}, C_{5}\right\} .
$$

The associated tensor basis is defined as :

$$
\begin{gathered}
{\left[\left[E^{(1)}\right]\right]=[p] \otimes[p],\left[\left[E^{(2)}\right]\right]=\frac{1}{2}[q] \otimes[q],\left[\left[E^{(3)}\right]\right]=\frac{1}{\sqrt{2}}[p] \otimes[q],} \\
{\left[\left[E^{(4)}\right]\right]=\frac{1}{\sqrt{2}}[q] \otimes[p],[[F]]=[q] \underline{\otimes}[q]-\left[\left[E^{(2)}\right]\right],[[G]]=[[I]]-\left[\left[E^{(1)}\right]\right]-\left[\left[E^{(2)}\right]\right]-[[F]],}
\end{gathered}
$$

wherein the two second-rank (idempotent) symmetric tensors $[p]$ and $[q]$ are defined by $[p]=\boldsymbol{n} \otimes \boldsymbol{n}$ and $[q]=[I]-[p],[I]$ and $[[I]]=[I] \underline{\mathbb{Q}}[I]$ are the second- and fourth-rank symmetric identity tensors respectively, and $\underline{\bar{Q}}$ denotes the usual symmetrized tensor product defined by $2([A] \underline{\bar{Q}}[B])_{i j k \ell}=[A]_{i k}[B]_{j \ell}+[A]_{i \ell}[B]_{j k}$ for any second-rank tensors $[A]$ and $[B]$. Note that this set of tensors does not form an orthogonal basis, and the associated multiplication table can be found in [48], pp. 164. The form taken by the deterministic (matrix) basis, using Kelvin's form, is recalled in Appendix A for $\boldsymbol{n}=(0,0,1)$. 
The a.s. positive-definiteness of random elasticity matrix $[\boldsymbol{C}]$ yields the following conditions on the random coordinates:

$$
\begin{gathered}
C_{1}+C_{2}>0, \\
C_{1} C_{2}-C_{3}^{2}>0, \\
C_{4}>0, \\
C_{5}>0 .
\end{gathered}
$$

A combination of Eqs. (28) and (29) implies that random components $C_{1}$ and $C_{2}$ are both strictly positive, so that support $\mathcal{S}$ can finally be written as $\mathcal{S}=\mathcal{S}_{123} \times \mathcal{S}_{4} \times \mathcal{S}_{5}$, with $\mathcal{S}_{4}=\mathcal{S}_{5}=\mathbb{R}^{+}$and

$$
\mathcal{S}_{123}=\left\{\left(c_{1}, c_{2}, c_{3}\right) \in \mathbb{R}^{+} \times \mathbb{R}^{+} \times \mathbb{R} \text { such that } \zeta\left(c_{1}, c_{2}, c_{3}\right)>0\right\},
$$

in which the mapping $\zeta: \mathbb{R}^{+} \times \mathbb{R}^{+} \times \mathbb{R} \rightarrow \mathbb{R}$ is defined as $\zeta\left(c_{1}, c_{2}, c_{3}\right)=c_{1} c_{2}-c_{3}{ }^{2}$. The graph of the limit surface $\zeta\left(c_{1}, c_{2}, c_{3}\right)=0$ is displayed in Fig. 1. Furthermore, closed-form expressions for the random

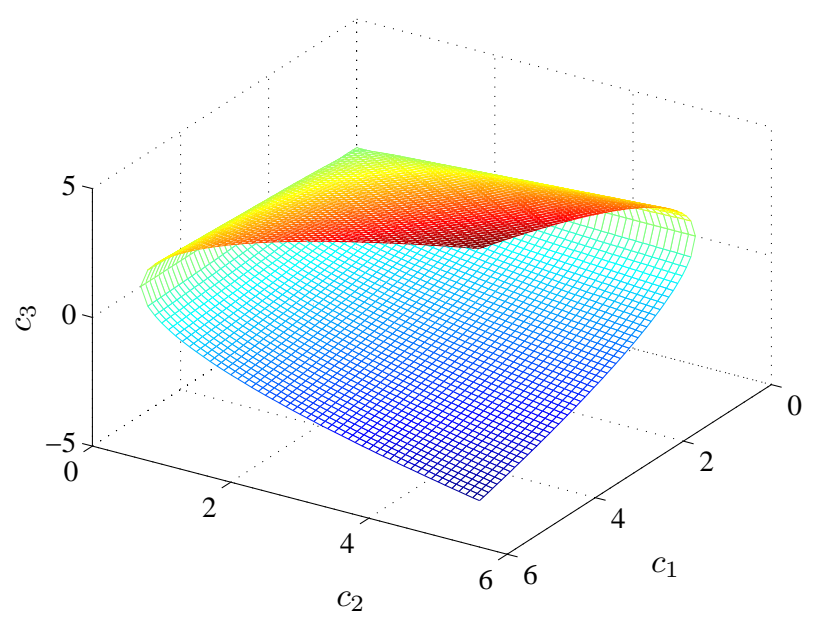

Figure 1: Graph of the limit surface $\zeta\left(c_{1}, c_{2}, c_{3}\right)=0$.

eigenvalues can be readily obtained and mapping $\varphi$, introduced in Section 3.1, turns out to be defined as:

$$
\varphi(\boldsymbol{c})=\log \left(c_{4}^{2} c_{5}^{2}\left(c_{1} c_{2}-c_{3}^{2}\right)\right) .
$$

From Eqs. (11) and (33), we then deduce that the p.d.f. $p_{\boldsymbol{C}}$ of random vector $\boldsymbol{C}$ writes

$$
\begin{aligned}
p_{\boldsymbol{C}}(\boldsymbol{c})= & \mathbb{1}_{\mathcal{S}_{123}}\left(\left(c_{1}, c_{2}, c_{3}\right)\right) k_{123} \exp \left\{-\sum_{i=1}^{3} \lambda_{\mathrm{sol} i}^{(1)} c_{i}-\lambda_{\mathrm{sol}}^{(2)} \log \left(c_{1} c_{2}-c_{3}{ }^{2}\right)\right\} \\
& \times \mathbb{1}_{\mathbb{R}^{+}}\left(c_{4}\right) k_{4} c_{4}{ }^{-2 \lambda_{\mathrm{sol}}^{(2)}} \exp \left\{-\lambda_{\mathrm{sol} 4}^{(1)} c_{4}\right\} \\
& \times \mathbb{1}_{\mathbb{R}^{+}}\left(c_{5}\right) k_{5} c_{5}{ }^{-2 \lambda_{\mathrm{sol}}^{(2)}} \exp \left\{-\lambda_{\mathrm{sol} 5}^{(1)} c_{5}\right\}
\end{aligned}
$$

in which $k_{123}, k_{4}$ and $k_{5}$ are new normalization constants. From Eq. (34), it can then be deduced that: 
(i) The random variables $C_{4}$ and $C_{5}$ are statistically independent from all other random variables and are Gamma distributed, $C_{4} \hookrightarrow \mathcal{G}\left(\alpha_{4}, \beta_{4}\right)$ and $C_{5} \hookrightarrow \mathcal{G}\left(\alpha_{5}, \beta_{5}\right)$, with parameters $\alpha_{4}=\alpha_{5}=1-2 \lambda_{\text {sol }}^{(2)}$, $\beta_{4}=1 / \lambda_{\mathrm{sol} 4}^{(1)}$ and $\beta_{5}=1 / \lambda_{\mathrm{sol} 5}^{(1)}$. It follows that the normalization constants read

$$
k_{4}=\lambda_{\mathrm{sol} 4}^{(1)}{ }^{1-2 \lambda_{\mathrm{sol}}^{(2)}} / \Gamma\left(1-2 \lambda_{\mathrm{sol}}^{(2)}\right) \text { and } k_{5}=\lambda_{\mathrm{sol} 5}^{(1)}{ }^{1-2 \lambda_{\mathrm{sol}}^{(2)}} / \Gamma\left(1-2 \lambda_{\mathrm{sol}}^{(2)}\right),
$$

wherein $x \mapsto \Gamma(x)$ represents the gamma function defined as $\Gamma(x)=\int_{0}^{\infty} t^{x-1} \exp \{-t\} d t$, and that

$$
\mathrm{E}\left\{C_{4}\right\}=\frac{1-2 \lambda_{\mathrm{sol}}^{(2)}}{\lambda_{\mathrm{sol} 4}^{(1)}} \text { and } \mathrm{E}\left\{C_{5}\right\}=\frac{1-2 \lambda_{\mathrm{sol}}^{(2)}}{\lambda_{\mathrm{sol} 5}^{(1)}}
$$

Thus, the value of Lagrange multipliers $\lambda_{\mathrm{sol} 4}^{(1)}$ and $\lambda_{\mathrm{sol} 5}^{(1)}$ can be readily deduced as soon as the mean values of $C_{4}$ and $C_{5}$ and Lagrange multiplier $\lambda_{\text {sol }}^{(2)}$ are fixed, hence reducing the size of the general optimization problem (24). Note that while we will not make use of this property in the following, in order to test the optimization algorithm, one may take advantage of similar reductions for larger values of parameter $N$.

Finally, the coefficient of variation of $C_{4}$ and $C_{5}$ is found to be $1 /\left(1-2 \lambda_{\text {sol }}^{(2)}\right)^{1 / 2}$, showing that random coordinates $C_{4}$ and $C_{4}$ exhibit exactly the same level of statistical fluctuations. The graph of mapping $\lambda^{(2)} \mapsto 1 /\left(1-2 \lambda^{(2)}\right)^{1 / 2}$ is displayed in Fig. 2 .

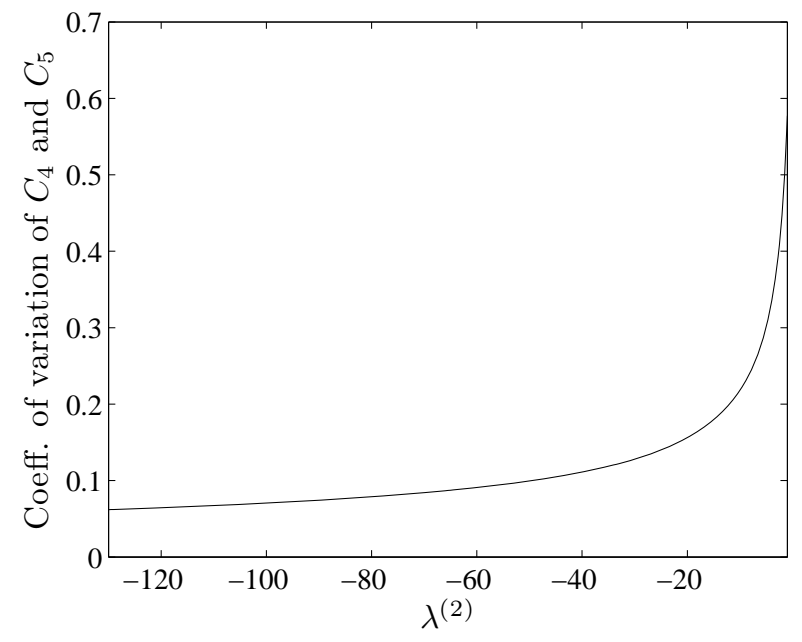

Figure 2: Graph of the coefficient of variation for random variables $C_{4}$ and $C_{5}$ as a function of Lagrange multiplier $\lambda^{(2)}$.

(ii) The random variables $C_{1}, C_{2}$ and $C_{3}$ are jointly distributed according to the p.d.f. $p_{C_{1}, C_{2}, C_{3}}$, defined 
from $\mathcal{S}_{123}$ into $\mathbb{R}^{+}$and given by:

$$
p_{C_{1}, C_{2}, C_{3}}\left(c_{1}, c_{2}, c_{3}\right)=\mathbb{1}_{\mathcal{S}_{123}}\left(\left(c_{1}, c_{2}, c_{3}\right)\right) k_{123} \exp \left\{-\sum_{i=1}^{3} \lambda_{\mathrm{sol} i}^{(1)} c_{i}-\lambda_{\mathrm{sol}}^{(2)} \log \left(c_{1} c_{2}-c_{3}{ }^{2}\right)\right\} .
$$

It is interesting to notice that the dependence structure induced by the MaxEnt procedure would be different (yielding four jointly distributed random components and one random component independent from all others), should a parametrization based on the non-zero components of the matrix form be retained. However, note that one would end up with the same structure making use of a straightforward change of variable.

For this application, we consider the following mean value, corresponding to a typical value for an unidirectional Carbon-Epoxy composite and exhibiting transverse isotropy with respect to $\boldsymbol{n}=(0,0,1)$ :

$$
[\underline{C}]=\left(\begin{array}{cccccc}
10.0735 & 0.5497 & 2.9745 & 0 & 0 & 0 \\
& 10.0735 & 2.9745 & 0 & 0 & 0 \\
& & 182.6657 & 0 & 0 & 0 \\
& & & 14 & 0 & 0 \\
& \text { Sym. } & & & 14 & 0 \\
& & & & & 9.5238
\end{array}\right),
$$

From Eq. (38) and the matrix expression of the basis (see Appendix A), it can be deduced that:

$$
\underline{c}_{1}=182.6657, \underline{c}_{2}=10.6232, \underline{c}_{3}=4.2066, \underline{c}_{4}=9.5238, \underline{c}_{5}=14 .
$$

In order to determine an admissible value for parameter $\nu_{C}$, the random matrix ensemble $S E^{+}$, introduced in [37] [38], is used. More specifically, we consider the mean value given by Eq. (38) and the mapping $\delta_{\boldsymbol{C}} \mapsto \nu_{\boldsymbol{C}}\left(\delta_{\boldsymbol{C}}\right)$ (see Eq. (1)) is constructed using numerical Monte Carlo simulations. The plot of $\delta_{\boldsymbol{C}} \mapsto$ $\nu_{C}\left(\delta_{\boldsymbol{C}}\right)$ is shown in Fig. 3. Considering a reasonable level of fluctuations, the value of parameter $\nu_{\boldsymbol{C}}$ is set to 17.3 , yielding the following expression for the target vector:

$$
\boldsymbol{f}^{\mathrm{target}}=(182.6657,10.6232,4.2066,9.5238,14,17.3) .
$$

\subsubsection{Computation of the Lagrange multipliers.}

The admissible spaces for the Lagrange multipliers are first determined using mathematical equivalents and imposing the integrability of the p.d.f. defined by Eq. (11) at both the origin and infinity. It can then be proven that:

$$
\mathcal{A}_{\boldsymbol{\lambda}(1)}=\left\{\left(\lambda_{1}^{(1)}, \ldots, \lambda_{5}^{(1)}\right) \in \mathbb{R}^{+} \times \mathbb{R}^{+} \times \mathbb{R} \times \mathbb{R}^{+} \times \mathbb{R}^{+} \text {such that } 2 \sqrt{\lambda_{1}^{(1)} \lambda_{2}^{(1)}}-\lambda_{3}^{(1)}>0\right\},
$$

and

$$
\left.\mathcal{A}_{\lambda^{(2)}}=\right]-\infty, 1 / 2[\text {. }
$$

Note that the definition of $\mathcal{A}_{\boldsymbol{\lambda}^{(1)}}$ and $\mathcal{A}_{\lambda^{(2)}}$ is consistent with the constraints associated with the definition

of parameters $\alpha_{4}, \alpha_{5}, \beta_{4}$, and $\beta_{5}$ for the Gamma distributed random variables $C_{4}$ and $C_{5}$. Following Section 3.2.3, expressions for the initial guess $\boldsymbol{\lambda}_{0}^{(1)}$ of $\boldsymbol{\lambda}^{(1)}$ are further obtained as:

$$
\lambda_{0}^{(1)}=\left(-\frac{\lambda_{0}^{(2)} \underline{c}_{2}}{\underline{c}_{1} \underline{c}_{2}-\underline{c}_{3}^{2}},-\frac{\lambda_{0}^{(2)} \underline{c}_{1}}{\underline{c}_{1} \underline{c}_{2}-\underline{c}_{3}^{2}}, 2 \frac{\lambda_{0}^{(2)} \underline{c}_{3}}{\underline{c}_{1} \underline{c}_{2}-\underline{c}_{3}^{2}},-2 \frac{\lambda_{0}^{(2)}}{\underline{c}_{4}},-2 \frac{\lambda_{0}^{(2)}}{\underline{c}_{5}}\right) .
$$




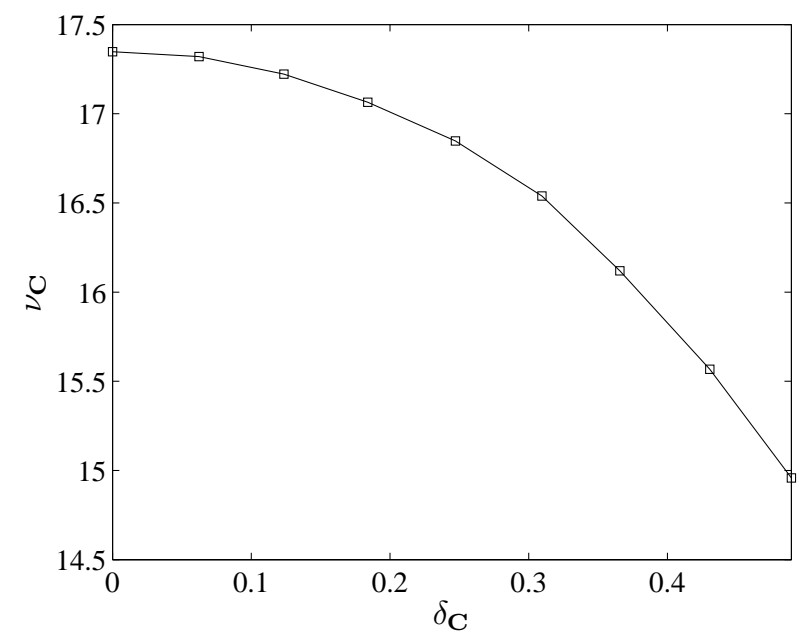

Figure 3: Graph of $\delta_{\boldsymbol{C}} \mapsto \nu_{\boldsymbol{C}}\left(\delta_{\boldsymbol{C}}\right)$ for $[\boldsymbol{C}] \in S E^{+}$with mean parameter $[\underline{C}]$.

wherein $\lambda_{0}^{(2)}$ denotes the initial guess for multiplier $\lambda^{(2)}$. A reasonable initial guess for $\lambda_{0}^{(2)}$ can be inferred from a parametric study, and it can be shown that setting $\lambda_{0}^{(2)}=-110$ yields a rather small initial value for the cost function $\mathcal{J}$. From Eq. (47), we then deduce the following initial guess:

$$
\boldsymbol{\lambda}_{0}^{(1)}=(0.6077,10.45,-0.4813,23.1,15.7143), \lambda_{0}^{(2)}=-110 .
$$

The ISDE has been discretized using the explicit Euler scheme considered in [41] and recalled in Appendix B. The convergence of the optimization algorithm (for which parameter $\alpha$ is set to 0.5; see Eq. (25)) is shown in Fig. 4, where the plot of the best (minimal) value for cost function $\mathcal{J}$ at iteration 'iter' is reported. A very good convergence of the algorithm is observed for a reasonably small number of iterations, hence validating the strategy for determining the prior guesses. The convergence to the stationary solution of the ISDE has been characterized using the mapping $M \mapsto \operatorname{convMES}(M)$, defined as

$$
\operatorname{convMES}(M)=M^{-1} \sum_{i=1}^{M}\left\|\boldsymbol{U}^{i}(\theta)\right\|^{2}
$$

and whose graph is shown in Fig. 5 (for the initial guess). The mathematical expectations have been estimated using the ergodic method, and the final sampling index $M$ has been determined from a convergence analysis on the mapping $M \mapsto \operatorname{convERG(} M)$ given by:

$$
\operatorname{convERG}(M)=\left\|\left(M-M_{0}+1\right)^{-1} \sum_{i=M_{0}}^{M} \boldsymbol{g}\left(\boldsymbol{U}^{i}(\theta)\right)\right\| .
$$

The graph of $M \mapsto \operatorname{convERG(} M)$ is plot in Fig. 6 (for the initial guess). From these figures, it follows that setting $M_{0}=3 \times 10^{5}$ and $M=1.1 \times 10^{6}$ yields reasonably converged results and these values are 




Figure 4: Convergence of the optimization algorithm: graph of iter $\mapsto$ convALG(iter).

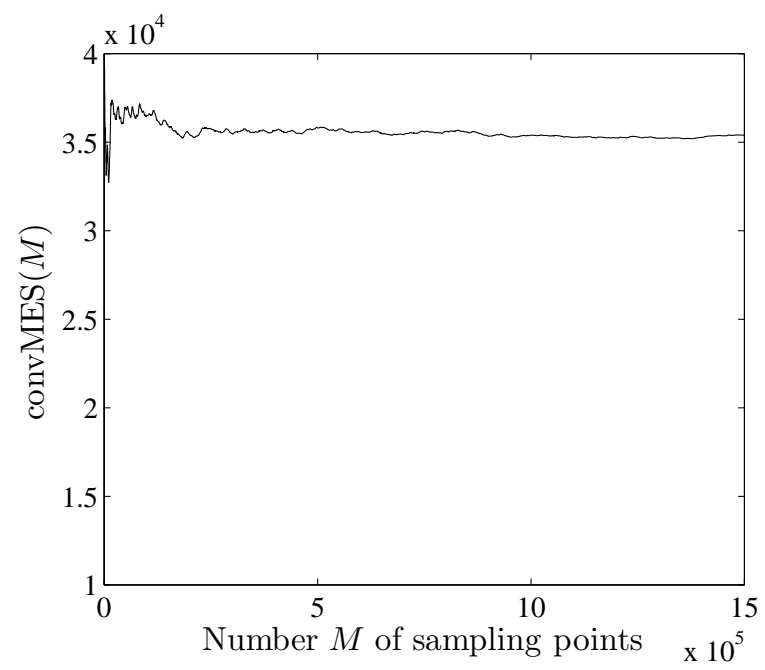

Figure 5: Convergence to the stationary solution of the ISDE: graph of $M \mapsto \operatorname{convMES}(M)$.

therefore used for the present study. Moreover, the values of parameters $f_{0}$ and $\Delta r$ are deduced from a preprocessing analysis and are set to 1 and 0.1 respectively. The optimal Lagrange multipliers $\boldsymbol{\lambda}_{\text {sol }}^{(1)}$ and $\lambda_{\text {sol }}^{(2)}$ are found to be

$$
\boldsymbol{\lambda}_{\mathrm{sol}}^{(1)}=(0.6142,10.5644,-0.4852,23.2096,15.7301), \lambda_{\mathrm{sol}}^{(2)}=-109.4626 .
$$




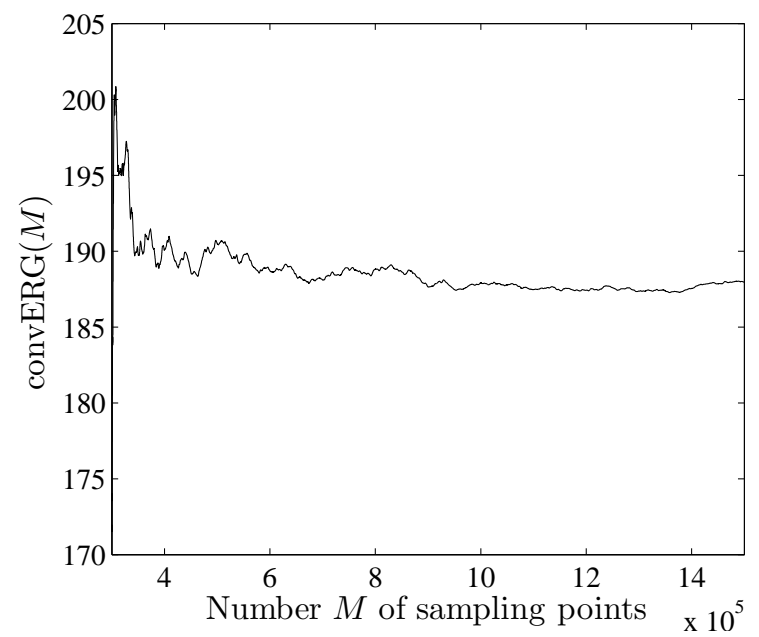

Figure 6: Convergence of the ergodic estimator: graph of $M \mapsto \operatorname{convERG(}(M)$.

for which the cost function $\mathcal{J}\left(\boldsymbol{\lambda}_{\text {sol }}\right)$ is equal to $1.4474 \cdot 10^{-4}$. The ergodic estimator $\boldsymbol{f}^{\text {erg }}$ of constraints vector yields:

$$
\boldsymbol{f}^{\mathrm{erg}}\left(\boldsymbol{\lambda}_{\mathrm{sol}}\right)=(182.6656,10.6214,4.2067,9.5197,13.9973,17.3155),
$$

which has to be compared with reference (40). It is seen that the estimated value matches the target value very well, with a maximum relative error of about $0.09 \%$. Note that the estimation of the mean values for $C_{4}$ and $C_{5}$, using Eqs. (36) and (47), yields 9.4756 and 13.9812, which has to be compared with the target values 9.5238 and 14 .

For the sake of completeness, the vector of constraints estimated by the Monte Carlo method has also been computed. The convergence of the statistical estimator is first characterized using the function $n_{s} \mapsto \operatorname{convMC}\left(n_{s}\right)$ defined as

$$
\operatorname{convMC}\left(n_{s}\right)=\left\|n_{s}{ }^{-1} \sum_{i=1}^{n_{s}} \boldsymbol{g}\left(\boldsymbol{C}\left(\theta_{i}\right)\right)\right\|
$$

and displayed in Fig. 7. It is seen that the convergence is reasonably reached for $n_{s}=500$ simulations, and the estimated vector of constraints is equal to

$$
\boldsymbol{f}^{\mathrm{mc}}\left(\boldsymbol{\lambda}_{\mathrm{sol}}\right)=(181.5684,10.7704,4.2621,9.5031,14.0728,17.3312),
$$

which is still in good agreement with the reference value, the maximum relative error being of about $1.38 \%$.

Remark concerning the calculation of $\mathrm{E}\left\{\mathbb{1}_{\mathcal{S}}\left(\boldsymbol{B}_{\boldsymbol{\lambda}}\right)\right\}$. For the presented calculations, it is observed that the statistical estimator of mathematical expectation $\mathrm{E}\left\{\mathbb{1}_{\mathcal{S}}\left(\boldsymbol{B}_{\boldsymbol{\lambda}}\right)\right\}$ is equal to 1 . In other words, the random generator does not draw samples that fall out of the support $\mathcal{S}$, neither in the transient nor in the stationary regime. This interesting behavior can be explained by noticing that (i) the mean values lie 


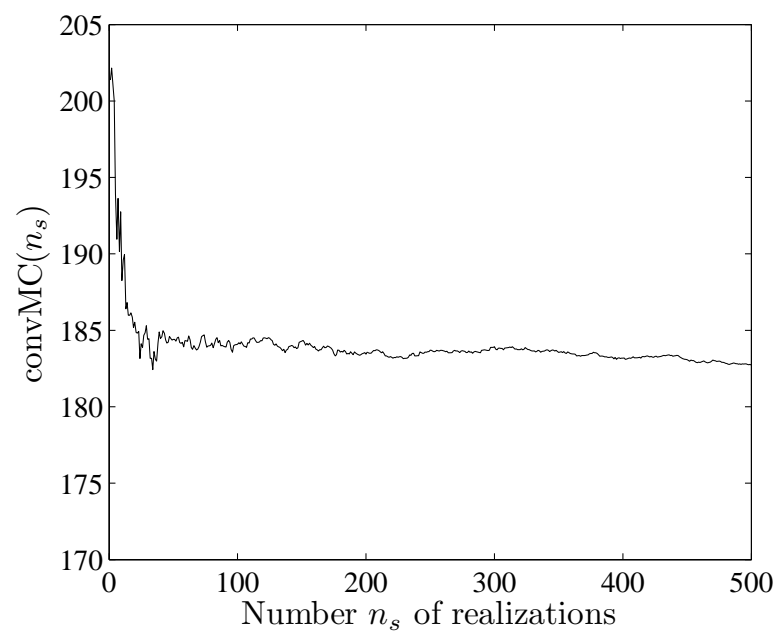

Figure 7: Convergence of the estimator based on the Monte Carlo method: graph of $n_{s} \mapsto \operatorname{convMC}\left(n_{s}\right)$.

pretty far from the boundary of the admissible space, with a level of fluctuations that is not too large; and that (ii) the form of the potential function and the repulsive log-condition (see Eq. (9)) make the chain be trapped in the correct attractive region (corresponding to the invariant measure under consideration) that is reached because of the good prior guess for the Lagrange multipliers.

Remark about computation time. The CPU time for solving the optimization problem is 112 seconds on a laptop (with one $1.4 \mathrm{GHz}$ single-core processor), making use of $\mathrm{C} / \mathrm{C}++$ (for the random generator) and Matlab (for the optimization algorithm) programming. The CPU time for randomly generating $n_{s}=500$ samples, with $M=3 \times 10^{5}$, is about 123 seconds using a single-core processor.

\section{GENERALIZED STOCHASTIC MODELING OF ELASTIC- ITY RANDOM MATRICES}

In this section, we make use of the previous derivations and propose a generalized stochastic model for random elasticity matrices that allows, by introducing an additional source of random anisotropy, for the prescription of a mean stochastic anisotropy measure, together with and apart from the level of statistical fluctuations (see [44] for similar considerations in the isotropic case).

\subsection{Overview of the construction}

Taking inspiration from the algebraic representation introduced in the generalized approach for random uncertainties derived in [42] (see also [44]), let the random elasticity matrix $[\boldsymbol{C}]$ be written as

$$
\left[\boldsymbol{C}^{\mathrm{g}}\right]=\left[\boldsymbol{L}^{\mathrm{sym}}\right][\boldsymbol{G}]\left[\boldsymbol{L}^{\mathrm{sym}}\right],
$$


in which the random matrices $\left[\boldsymbol{L}^{\mathrm{sym}}\right]$ and $[\boldsymbol{G}]$ satisfy the following properties:

$\left(P_{1}\right)\left[\boldsymbol{L}^{\mathrm{sym}}\right]$ is a $\mathbb{E} \mathrm{la}^{\mathrm{sym}}$-valued random variable defined as the almost-sure positive-definite square root of random matrix $\left[\boldsymbol{C}^{\mathrm{sym}}\right],\left[\boldsymbol{C}^{\mathrm{sym}}\right]=\left[\boldsymbol{L}^{\mathrm{sym}}\right]^{2}$, wherein $\left[\boldsymbol{C}^{\mathrm{sym}}\right]$ belongs to the random matrix ensemble defined in Section 3 and is such that $\mathrm{E}\left\{\left[\boldsymbol{C}^{\text {sym }}\right]\right\}=[\underline{C}]$.

$\left(P_{2}\right)[\boldsymbol{G}]$ is a $\mathbb{M}_{n}^{+}(\mathbb{R})$-valued random variable satisfying $\mathrm{E}\{[\boldsymbol{G}]\}=\left[I_{n}\right],\left[I_{n}\right]$ being the $(n \times n)$ identity matrix, and such that $[\boldsymbol{G}]$ and $[\boldsymbol{G}]^{-1}$ are second-order random variables.

$\left(P_{3}\right)$ The random variables $\left[\boldsymbol{L}^{\mathrm{sym}}\right]$ and $[\boldsymbol{G}]$ are statistically independent.

Following the terminology introduced in Section 3.1.1, we denote by $\left\{L_{i}\right\}_{i=1}^{N}$ (resp. $\left\{C_{i}\right\}_{i=1}^{N}$ ) the random coordinates of $\left[\boldsymbol{L}^{\mathrm{sym}}\right]$ (resp. $\left[\boldsymbol{C}^{\mathrm{sym}}\right]$ ) on tensor basis $\left\{\left[E_{\mathrm{sym}}{ }^{(i)}\right]\right\}_{i=1}^{N}$ of $\mathbb{E l a}^{\mathrm{sym}}$. Since $\left[\boldsymbol{C}^{\mathrm{sym}}\right]$ is positivedefinite almost surely, it follows that the almost-sure positive-definite random matrix $\left[\boldsymbol{L}^{\mathrm{sym}}\right]$ is uniquely defined (see Theorem 7.2.6 in pp. 405 of [16], for instance) through a given, component-wise transformation denoted by $\Psi^{\mathrm{sym}}=\left(\Psi^{\mathrm{sym}}{ }_{1}, \ldots, \Psi^{\mathrm{sym}}{ }_{N}\right)$ with $L_{i}=\Psi^{\mathrm{sym}}{ }_{i}\left(C_{1}, \ldots, C_{N}\right)$. The definition of such mappings can be found in [48] for all material symmetry classes (see Appendix C for the case of transversely isotropic materials) and allows the joint p.d.f. for random coefficients of $\left[\boldsymbol{L}^{\mathrm{sym}}\right]$ to be derived from the joint p.d.f. of coefficients of $\left[\boldsymbol{C}^{\mathrm{sym}}\right]$ (note however that this joint p.d.f. will not be provided for the application proposed below, taking into account the rather complicated algebraic form of the function).

From Eq. (51), it is readily seen that:

(i) If $\delta_{[G]}=0,\left[\boldsymbol{C}^{\mathrm{g}}\right]$ is a $\mathbb{E} \mathrm{la}^{\mathrm{sym}}$-valued random variable and the probabilistic model then coincides with the one defined in Section 3, generating realizations that belong to $\mathbb{E} l a^{\mathrm{sym}}$ almost surely.

(ii) If $\left[\boldsymbol{L}^{\text {sym }}\right]$ is deterministic (i.e. if $\lambda^{(2)} \rightarrow-\infty$ ), random variable $\left[\boldsymbol{C}^{\mathrm{g}}\right]$ exhibits anisotropic fluctuations around the symmetry class 'sym'. Note that the random matrix ensemble thus obtained does not coincide with the subset of $S E^{+}$for which the mean model exhibits the symmetry class 'sym', since $\left[\boldsymbol{L}^{\text {sym }}\right]$ is a (sparse) matrix that does not correspond to the (upper triangular) matrix involved in the Cholesky decomposition of $\left[\underline{C}^{\mathrm{sym}}\right]$.

(iii) If both $\left[\boldsymbol{L}^{\mathrm{sym}}\right]$ and $[\boldsymbol{G}]$ are random, realizations of $\left[\boldsymbol{C}^{\mathrm{g}}\right]$ present stochastic fluctuations which are more or less 'anisotropic' around the symmetry class 'sym'. Furthermore, the level of stochastic anisotropy mainly depends on parameter $\delta_{[G]}$, while the level of statistical fluctuation is controlled by both $\delta_{[\boldsymbol{G}]}$ and $\delta_{\left[\boldsymbol{L}^{\mathrm{sym}}\right]}$.

Consequently, for a given material symmetry class 'sym', the approach allows one to generate realizations whose anisotropy index, defined with respect to the distance to $\mathbb{E l a}{ }^{\text {sym }}$, can range from 0 to $+\infty$ (corresponding the triclinic class) almost surely.

Remark. Although there is an obvious analogy between the representation given by Eq. (51) and the one used in [44] for the isotropic class, it should be pointed out that the two approaches basically differ, for the isotropic symmetry, in the way the constraint of invertibility is introduced. In [44], the information regarding the invertibility condition is different from the one which has been considered in this work (as well as in the nonparametric probabilistic approach derived in [37] and [38]), yielding random moduli that exhibit exactly the same level of fluctuations. On the contrary, it is worth while to note that the coefficients of variations of the random bulk and shear moduli would be different (and equal to $1 / \sqrt{1-\lambda_{\text {sol }}^{(2)}}$ and $1 / \sqrt{1-5 \lambda_{\text {sol }}^{(2)}}$ respectively) in the proposed approach. 


\subsection{Probability distribution for the anisotropic stochastic germ $[G]$}

From property $\left(P_{2}\right)$, the random anisotropic germ $[\boldsymbol{G}]$ is assumed to belong to the normalized positivedefinite ensemble $S G^{+}$defined in [39]. This random matrix ensemble has been defined by using the MaxEnt principle, for which a log-condition similar to Eq. (9) and the mean constraint $\mathrm{E}\{[\boldsymbol{G}]\}=\left[I_{n}\right]$ have been considered. The p.d.f. $p_{[G]}$ then reads

$$
p_{[\boldsymbol{G}]}([G])=\mathbb{1}_{\mathbb{M}_{n}^{+}(\mathbb{R})}([G]) k_{[\boldsymbol{G}]} \operatorname{det}([G])^{(n+1)\left(1-\delta_{[\boldsymbol{G}]}{ }^{2}\right) /\left(2 \delta_{[\boldsymbol{G}]}^{2}\right)} \exp \left\{-\frac{n+1}{2 \delta_{[\boldsymbol{G}]}} \operatorname{tr}([G])\right\},
$$

in which $k_{[\boldsymbol{G}]}$ is the constant of normalization. The description of an efficient generator for random variable $[\boldsymbol{G}]$ can be found in [37], while fundamental properties and associated algebraic results can be found in [37], [38] and [39].

\subsection{Fundamental properties of random matrix $\left[C^{\mathrm{g}}\right]$}

The following properties for random elasticity matrix $\left[\boldsymbol{C}^{\mathrm{g}}\right]$ can be easily deduced.

Property 4.3.1 $\mathrm{E}\left[C^{g}\right]=[\underline{C}]$.

The result immediately follows from the definition of $\left[\boldsymbol{C}^{\mathrm{g}}\right]$, invoking the independence of $\left[\boldsymbol{L}^{\mathrm{sym}}\right]$ and $[\boldsymbol{G}]$ and making use of $\mathrm{E}\left\{\left[\boldsymbol{C}^{\text {sym }}\right]\right\}=[\underline{C}]$, together with $\mathrm{E}\{[\boldsymbol{G}]\}=\left[I_{n}\right]$.

Property 4.3.2 $\left[\boldsymbol{C}^{\mathrm{g}}\right]$ is a second-order random variable.

Since $\|[C]\| \leq\|[C]\|_{\mathrm{F}} \leq \sqrt{n}\|[C]\|$ for all $[C]$ in $\mathbb{M}_{n}^{+}(\mathbb{R})$ and from Eq. (51) and $\left(P_{3}\right)$, it can be deduced that $\mathrm{E}\left\{\left\|\left[\boldsymbol{C}^{\mathrm{g}}\right]\right\|_{\mathrm{F}}^{2}\right\} \leq n \mathrm{E}\left\{\left\|\left[\boldsymbol{L}^{\text {sym }}\right]\right\|^{4}\right\} \mathrm{E}\left\{\|[\boldsymbol{G}]\|^{2}\right\}$. Since $\left\|\left[\boldsymbol{L}^{\text {sym }}\right]\right\|=\left\|\left[\boldsymbol{C}^{\text {sym }}\right]\right\|^{1 / 2}$ and since $\left[\boldsymbol{C}^{\text {sym }}\right]$ is a second-order random variable, it follows that $\mathrm{E}\left\{\left\|\left[\boldsymbol{L}^{\text {sym }}\right]\right\|^{4}\right\} \leq \mathrm{E}\left\{\left\|\left[\boldsymbol{C}^{\text {sym }}\right]\right\|_{\mathrm{F}}^{2}\right\}<+\infty$. From $\left(P_{2}\right)$, one has $\mathrm{E}\left\{\|[\boldsymbol{G}]\|^{2}\right\}<$ $\mathrm{E}\left\{\|[\boldsymbol{G}]\|_{\mathrm{F}}^{2}\right\}<+\infty$ and consequently, we deduce that $\mathrm{E}\left\{\left\|\left[\boldsymbol{C}^{\mathrm{g}}\right]\right\|_{\mathrm{F}}^{2}\right\}<+\infty$.

Property 4.3.3 $\left[\boldsymbol{C}^{\mathrm{g}}\right]^{-1}$ is a second-order random variable.

Similarly, one has $\mathrm{E}\left\{\left\|\left[\boldsymbol{C}^{\mathrm{g}}\right]^{-1}\right\|_{\mathrm{F}}^{2}\right\} \leq n \mathrm{E}\left\{\left\|\left[\boldsymbol{L}^{\text {sym }}\right]^{-1}\right\|^{4}\right\} \mathrm{E}\left\{\left\|[\boldsymbol{G}]^{-1}\right\|^{2}\right\}$ and the result follows from $\left(P_{1}\right)$ and $\left(P_{2}\right)$.

\subsection{Application of the generalized approach to the class of transversely isotropic tensors}

In this section, we demonstrate the capabilities of the generalized probabilistic approach for the modeling of random media, for which the material symmetry constraint is defined with respect to the transversely isotropic class. The mean model is defined by Eq. (38) and four levels of statistical fluctuations are considered for each random matrix, that are:

- $\delta_{[\boldsymbol{G}]}=0, \delta_{[\boldsymbol{G}]}=0.1, \delta_{[\boldsymbol{G}]}=0.2, \delta_{[\boldsymbol{G}]}=0.3$

- $\delta_{\left[\boldsymbol{L}^{\mathrm{sym}}\right]}=0, \delta_{\left[\boldsymbol{L}^{\mathrm{sym}}\right]}\left(\nu_{\boldsymbol{C}}=17.3\right), \delta_{\left[\boldsymbol{L}^{\mathrm{sym}}\right]}\left(\nu_{\boldsymbol{C}}=17.1\right), \delta_{\left[\boldsymbol{L}^{\mathrm{sym}}\right]}\left(\nu_{\boldsymbol{C}}=16.9\right)$,

where the notation $\delta_{\left[\boldsymbol{L}^{\text {sym }}\right]}\left(\nu_{C}=\gamma\right),|\gamma|<+\infty$, denotes the level of fluctuations, estimated using mathematical statistics, exhibited by random variable $\left[\boldsymbol{L}^{\text {sym }}\right]$ defined as the positive-definite square root of 
random matrix $\left[\boldsymbol{C}^{\text {sym }}\right]$ whose probability distribution is parametrized by the Lagrange multipliers such that the log-condition (see Eq. (9)) is satisfied with parameter $\gamma$ as the r.h.s.

The very first step of the methodology therefore involves the computation of such multipliers, this task being completed following the methodology introduced in Section 3.2.3 and exemplified in Section 3.3.2. For the two highest levels of fluctuations $\delta_{\left[\boldsymbol{L}^{\text {sym }}\right]}\left(\nu_{\boldsymbol{C}}=17.1\right)$ and $\delta_{\left[\boldsymbol{L}^{\text {sym }}\right]}\left(\nu_{\boldsymbol{C}}=16.9\right)$, the optimization algorithm is initialized by respectively setting:

- $\lambda_{0}^{(2)}=-10, f_{0}=0.3, \Delta r=0.1$

- $\lambda_{0}^{(2)}=-6.5, f_{0}=0.24, \Delta r=0.1$.

The convergence analysis regarding the solution of the ISDE and the ergodic estimator is performed (see the graphs of $M \mapsto \operatorname{convMES}(M)$ and $M \mapsto \operatorname{convERG(} M)$ in Fig. 13, appendix D, for the second case) and shows that for such levels of statistical fluctuations, the convergence is obtained for $M_{0}=3 \times 10^{6}$ and $M=7 \times 10^{6}$. The graph of iter $\mapsto$ convALG(iter) is shown in Fig. 14, appendix D, for $\nu_{C}=17.1$ and $\nu_{C}=16.9$. A reasonably fast convergence of the optimization algorithm is observed, the solving procedure typically requiring less than 20 iterations. The levels of fluctuations associated with the logcondition defined above are then found to be $\delta_{\left[\boldsymbol{L}^{\text {sym }}\right]}=0, \delta_{\left[\boldsymbol{L}^{\mathrm{sym}}\right]}=0.05, \delta_{\left[\boldsymbol{L}^{\mathrm{sym}}\right]}=0.15$ and $\delta_{\left[\boldsymbol{L}^{\mathrm{sym}}\right]}=0.2$.

The plots of the marginal probability density functions $c_{i} \mapsto p_{C_{i}}\left(c_{i}\right), i=1, \ldots, 5$, estimated using 1000 independent realizations, are shown in Figs. 8, 9 and 10.
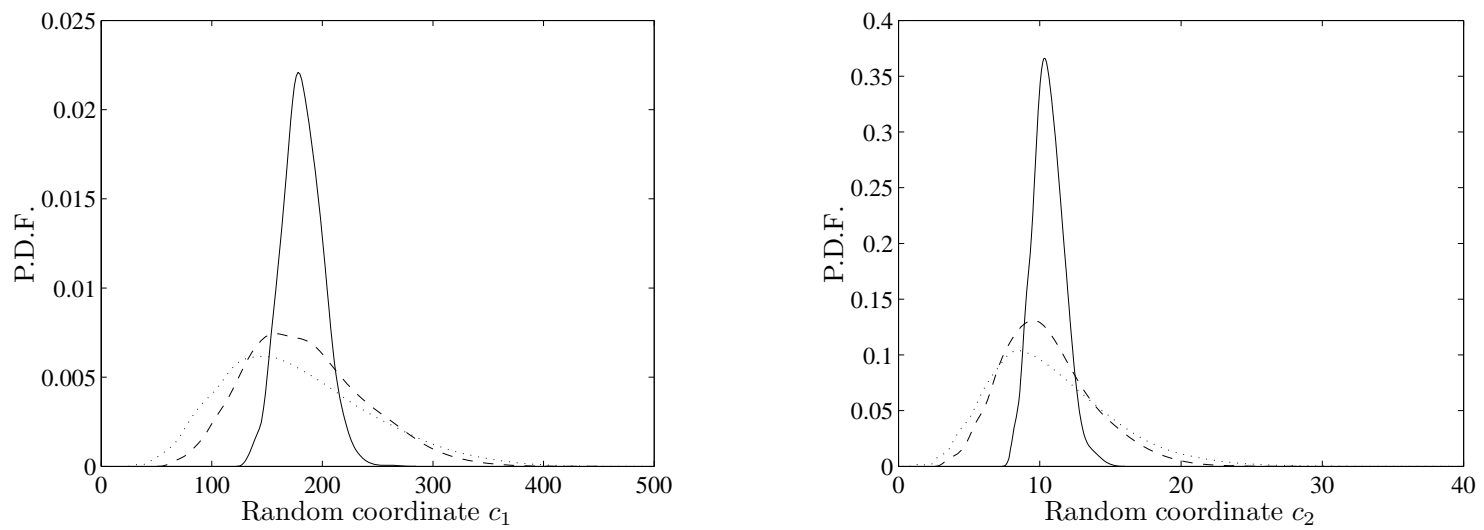

Figure 8: Graph of the p.d.f for random variables $C_{1}$ (left) and $C_{2}$ (right) for $\delta_{\left[\boldsymbol{L}^{\text {sym }}\right]}=0.05$ (solid line), $\delta_{\left[\boldsymbol{L}^{\text {sym }}\right]}=0.15$ (dashed line) and $\delta_{\left[\boldsymbol{L}^{\text {sym }}\right]}=0.2($ dotted line $)$.

The influence of parameters $\delta_{\left[\boldsymbol{L}^{\text {sym }}\right]}$ and $\delta_{[\boldsymbol{G}]}$ on the overall level of statistical fluctuations $\delta_{\left[\boldsymbol{C}^{g}\right]}$ is characterized in Fig. 11, where the graph of mapping $\left(\delta_{\left[\boldsymbol{L}^{\mathrm{sym}}\right]}, \delta_{[\boldsymbol{G}]}\right) \mapsto \delta_{\left[\boldsymbol{C}^{g}\right]}$ is depicted.

It is seen that both $\delta_{\left[\boldsymbol{L}^{\text {sym }}\right]}$ and $\delta_{[\boldsymbol{G}]}$ have an influence on $\delta_{\left[\boldsymbol{C}^{g}\right]}$, which increases with respect to the two parameters. Note that while $\delta_{\left[\boldsymbol{L}^{\text {sym }}\right]}$ seems to have a stronger influence in the proposed application, such a result may depend on the retained mean model.

In order to illustrate the capability of the generalized approach regarding the prescription of a mean stochastic anisotropy measure (to be defined), let us briefly discuss the definition of the projection of elasticity tensors with an arbitrary elasticity symmetry onto the set $\mathbb{E}$ la $^{\text {sym }}$. 

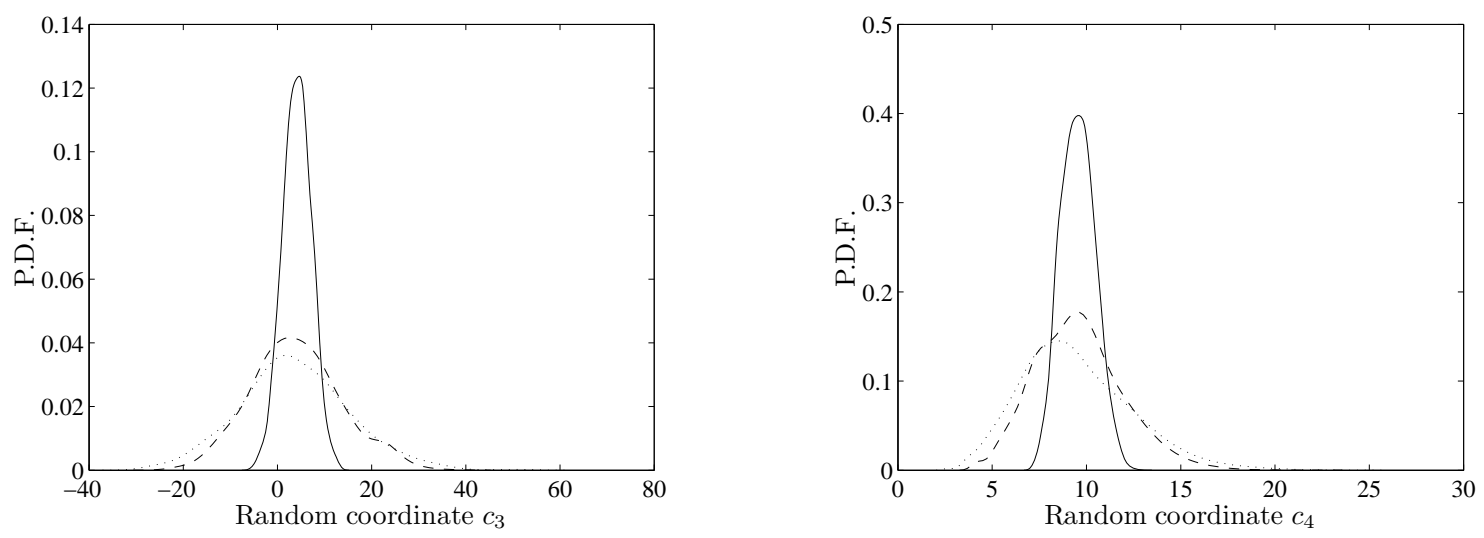

Figure 9: Graph of the p.d.f for random variables $C_{3}$ (left) and $C_{4}$ (right) for $\delta_{\left[\boldsymbol{L}^{\text {sym }}\right]}=0.05$ (solid line), $\delta_{\left[\boldsymbol{L}^{\mathrm{sym}}\right]}=0.15$ (dashed line) and $\delta_{\left[\boldsymbol{L}^{\mathrm{sym}}\right]}=0.2($ dotted line $)$.

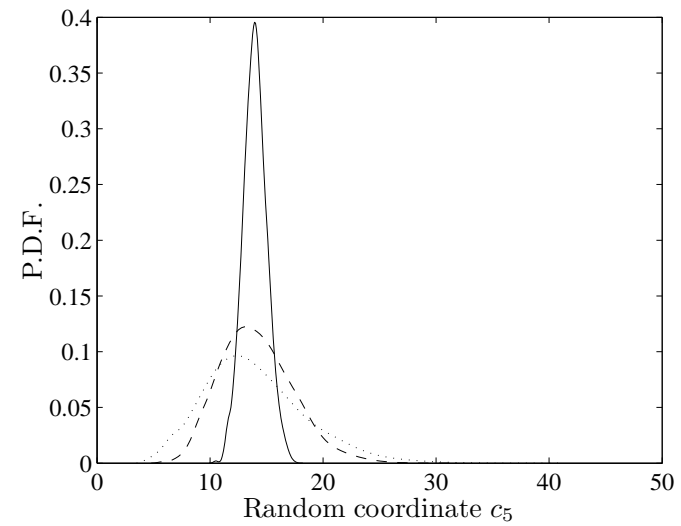

Figure 10: Graph of the p.d.f for random variable $C_{5}$ for $\delta_{\left[L^{\mathrm{sym}}\right]}=0.05$ (solid line), $\delta_{\left[L^{\mathrm{sym}}\right]}=0.15$ (dashed line) and $\delta_{\left[\boldsymbol{L}^{\mathrm{sym}}\right]}=0.2$ (dotted line).

Let $\mathrm{P}^{\mathrm{sym}}$ be the projection operator onto $\mathbb{E l a}{ }^{\mathrm{sym}}$. For any elasticity matrix $[C]$, we then denote by $[C]_{\text {proj }}^{\text {sym }}=\mathrm{P}^{\text {sym }}([C]) \in \mathbb{E} l^{\text {sym }}$ the associated projection, defined as

$$
[C]_{\text {proj }}^{\text {sym }}=\underset{[X] \in \mathbb{E l a}^{\text {sym }}}{\arg \min } \mathrm{d}([C]-[X]),
$$

in which d is any suitable metric in $\mathbb{E}$ la. In this work, we make use of the Euclidean distance, defined for any elasticity matrices $[C]_{1}$ and $[C]_{2}$ by:

$$
\mathrm{d}\left([C]_{1},[C]_{2}\right)=\left\|[C]_{1}-[C]_{2}\right\|_{\mathrm{F}} .
$$






Figure 11: Graph of the mapping $\left(\delta_{\left[\boldsymbol{L}^{\mathrm{sym}}\right]}, \delta_{[\boldsymbol{G}]}\right) \mapsto \delta_{\left[\boldsymbol{C}^{g}\right]}$.

Note that the definition and characterization of distances and associated projections have been extensively studied within the deterministic framework of theoretical elasticity: see [2] and [28] for discussions about distances in Ela; [29] and [4] for closed-form expressions of the projections expressed in matrix and vector forms respectively and [5] [21] [22] [23] for discussions regarding the definition of projections and closest approximations taking into account the symmetry reference frame. Physical and mathematical interpretations for the case of random elasticity matrices have been discussed quite recently in [12] [13] [14]. Consequently, such issues will not be addressed further in this paper and the interested reader is referred to the references given above (see the references therein as well).

We then introduce the $\mathbb{R}^{+}$-valued random variable $\mathcal{D}\left(\left[\boldsymbol{C}^{g}\right]\right)$, corresponding to a stochastic anisotropy measure and defined as the distance between $\left[\boldsymbol{C}^{g}\right]$ and its projection onto the set of transversely isotropic tensors (the material symmetry being defined with respect to a given unit normal $\boldsymbol{n}$ )

$$
\mathcal{D}\left(\left[\boldsymbol{C}^{g}\right]\right)=\mathrm{d}\left(\left[\boldsymbol{C}^{g}\right], \mathrm{P}^{\text {trans-iso }}\left(\left[\boldsymbol{C}^{g}\right]\right)\right),
$$

where $\mathrm{P}^{\text {trans-iso }}$ denotes the projection operator for the set $\mathbb{E}$ la $^{\text {trans-iso }}$ of transversely isotropic tensors (defined with respect to $\boldsymbol{n}$ ). Such a definition generalizes the usual concept of anisotropy measure, which is typically understood as the distance to the isotropic class. The mean value $\mathrm{E}\left\{\mathcal{D}\left(\left[\boldsymbol{C}^{g}\right]\right)\right\}$ of the anisotropy measure is worth characterizing, for it allows for the control (in the mean sense) of the distance to the considered (transversely isotropic) symmetry class. The graph of the mapping $\left(\delta_{\left[\boldsymbol{L}^{\text {sym }}\right]}, \delta_{[\boldsymbol{G}]}\right) \mapsto \mathrm{E}\left\{\mathcal{D}\left(\left[\boldsymbol{C}^{g}\right]\right)\right\}$ is shown in Fig. 12, with the mathematical expectation estimated from 1000 independent realizations.

It is readily seen that:

(i) For a given value of $\delta_{\left[\boldsymbol{L}^{\mathrm{sym}}\right]}$, the mean distance to the set of transversely isotropic tensors increases with parameter $\delta_{[\boldsymbol{G}]}$.

(ii) For a given value of $\delta_{[\boldsymbol{G}]}$ (controlling the anisotropic source of randomness), parameter $\delta_{\left[\boldsymbol{L}^{\text {sym }}\right]}$ has a negligible influence on the mean distance. 


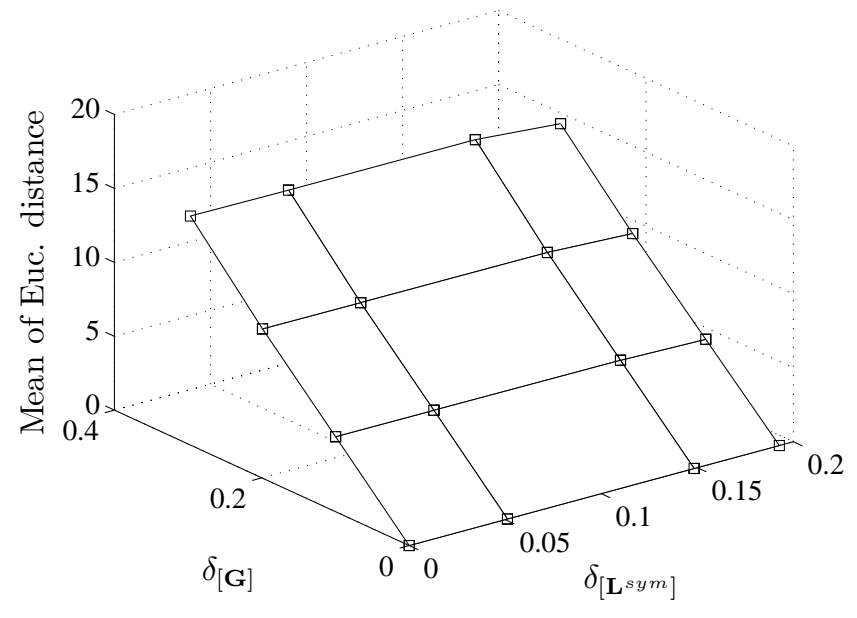

Figure 12: Graph of the mapping $\left(\delta_{\left[\boldsymbol{L}^{\mathrm{sym}}\right]}, \delta_{[\boldsymbol{G}]}\right) \mapsto \mathrm{E}\left\{\mathcal{D}\left(\left[\boldsymbol{C}^{g}\right]\right)\right\}$.

From Figs 11 and 12, it can then be deduced that the generalized probabilistic formulation given by Eq. (51) allows one to prescribe the mean distance to a material symmetry class, regardless of the overall level of statistical fluctuations exhibited by the random elasticity tensor.

\section{CONCLUSION}

In this paper, we addressed the construction of a stochastic representation for fourth-order random elasticity tensors, taking into account some properties related to material symmetries. Such a construction has been achieved in two steps, each of which being primarily concerned with a particular range of applicability.

First of all, we considered the construction of a probabilistic model for elasticity random matrices that exhibit some symmetry properties. Such an issue is of primary importance for many practical (engineering) situations, for which either experimental or numerical constraints may not allow for information related to anisotropic fluctuations to be integrated. Such limitations are for instance encountered in the modeling of wave propagation through random materials (e.g. for Structural Health Monitoring), where the consideration of weak symmetries significantly increases the computation time. In this context, we introduced a methodology relying on the decomposition of the stochastic elasticity tensor on a deterministic tensor basis. The probability distribution of the random coordinates thus defined is constructed invoking the MaxEnt principle, for which only fundamental and available information is considered. In particular, it is shown that the separability of the mapping involved in the log-condition generates a statistical dependence between some components of the elasticity tensor. It is worth noticing that such a dependence, which is intrinsic to the retained parametrization, does not come from an arbitrary, end-user's choice and that the result obtained here for the transversely isotropic class can be readily extended to all other classes. Based on the general form for the probability distribution, we further discussed strategies for random generation, with a special emphasis on the use of MCMC techniques when the dimension becomes quite large. A numerical strategy for the computation of the Lagrange multipliers is also proposed and the 
approach is exemplified in the case of a material that is transversely isotropic almost surely.

Then, we made use of such derivations to propose a generalized probabilistic model for random elasticity matrices that takes into account almost separately, by introducing an additional source of random anisotropy, constraints on both the level of stochastic anisotropy and the level of statistical fluctuations. Such a model, which is especially suitable for the modeling of random heterogenous microstructures at the mesoscale, can be interpreted within the framework of the generalized approach for random uncertainties defined in [42] and can be seen as the generalization of the model proposed in [44] (for the isotropic class) to all material symmetry classes. It is worth pointing out that such a generalization raises many issues, among which the computation of the multipliers and the construction of an efficient random generator (which are both straightforward in the isotropic case), which have been successfully addressed in this paper. An example is finally provided and demonstrates the efficiency of the approach.

The proposed generalized probabilistic approach is more general than the stochastic representations for elasticity tensors previously derived from a MaxEnt procedure in the literature, and allows for constraints related to material symmetries to be taken into account. The model may therefore pave the way for inverse identification and forward predictive simulations in high dimension when the underlying physics of uncertainty propagation turns out to be sensitive to symmetry properties.

\section{ACKNOWLEDGEMENTS}

This research was funded by the French Research Agency (Agence Nationale de la Recherche) under TYCHE contract ANR-2010-BLAN-0904.

\section{A MATRIX FORM BASIS FOR TRANSVERSELY ISOTROPIC TENSOR}

Denoting by $\left[0_{3}\right]$ the $(3 \times 3)$ zero matrix, the tensorial basis for transversely isotropic tensors, defined with respect to the axis vector $(0,0,1)$, is given by:

$$
\begin{aligned}
& {\left[E^{(1)}\right]=\left(\begin{array}{cccc}
0 & 0 & 0 & \\
0 & 0 & 0 & {\left[0_{3}\right]} \\
0 & 0 & 1 & \\
& {\left[0_{3}\right]} & & {\left[0_{3}\right]}
\end{array}\right),\left[E^{(2)}\right]=\left(\begin{array}{cccc}
0.5 & 0.5 & 0 & \\
0.5 & 0.5 & 0 & {\left[0_{3}\right]} \\
0 & 0 & 0 & \\
& & & \\
& & &
\end{array}\right)}
\end{aligned}
$$

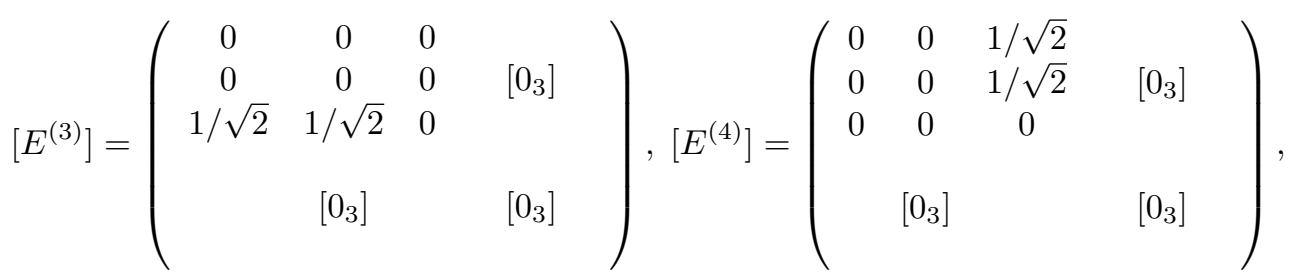




$$
[F]=\left(\begin{array}{cccccc}
0.5 & -0.5 & 0 & & & \\
-0.5 & 0.5 & 0 & & {\left[0_{3}\right]} & \\
0 & 0 & 0 & & & \\
& & & 0 & 0 & 0 \\
& {\left[0_{3}\right]} & & 0 & 0 & 0 \\
& & & 0 & 0 & 1
\end{array}\right),[G]=\left(\begin{array}{llll}
{\left[0_{3}\right]} & & & \\
& & & \\
& & & \\
& 1 & 0 & 0 \\
{\left[0_{3}\right]} & 0 & 1 & 0 \\
& 0 & 0 & 0
\end{array}\right)
$$

\section{B EXPLICIT EULER SCHEME FOR THE ISDE DISCRETIZA- TION}

For notational convenience, let $\boldsymbol{U}^{k}=\boldsymbol{U}\left(r_{k}\right)$ and $\boldsymbol{V}^{k}=\boldsymbol{V}\left(r_{k}\right)$. The explicit Euler scheme then reads [41]:

$$
k=1, \ldots, M-1,\left\{\begin{array}{l}
\boldsymbol{U}^{k+1}=\boldsymbol{U}^{k}+\Delta r \boldsymbol{V}^{k} \\
\boldsymbol{V}^{k+1}=\left(1-\frac{f_{0}}{2} \Delta r\right) \boldsymbol{V}^{k}+\Delta r \boldsymbol{L}^{k}+\sqrt{f_{0}} \Delta \boldsymbol{W}^{k+1},
\end{array}\right.
$$

where $\boldsymbol{U}^{1}=\boldsymbol{u}_{0}$ and $\boldsymbol{V}^{1}=\boldsymbol{v}_{0}, \boldsymbol{W}^{k+1}$ is a second-order Gaussian centered $\mathbb{R}^{N}$-valued random variable with covariance matrix $\Delta r\left[I_{N}\right]$ and $\boldsymbol{L}^{k}$ is a $\mathbb{R}^{N}$-valued random variable whose numerical approximation is defined component-wise as

$$
L^{k}{ }_{j}=-\frac{\Phi\left(\Delta \boldsymbol{U}^{k, j} ; \boldsymbol{\lambda}\right)-\Phi\left(\boldsymbol{U}^{k} ; \boldsymbol{\lambda}\right)}{U^{k+1}{ }_{j}-U^{k}}, j=1, \ldots, N,
$$

where $\Delta \boldsymbol{U}^{k, j}$ is a $\mathbb{R}^{N}$-valued random variable whose $m$-th component writes $\Delta U^{k, j}{ }_{m}=U^{k}{ }_{m}+\delta_{j m}\left(U^{k+1}{ }_{m}-\right.$ $\left.U^{k}{ }_{m}\right)$.

\section{DEFINITION OF MAPPING $\Psi^{\text {trans-iso }}$}

Making use of the multiplication table between the tensors of the basis for $\mathbb{E}^{\text {latans-iso }}{ }^{\text {, one has: }}$

$$
\left\{\begin{array}{l}
L_{1}=\left(C_{1}+\sqrt{C_{1} C_{2}-C_{3}^{2}}\right) / \xi \\
L_{2}=\left(C_{2}+\sqrt{C_{1} C_{2}-C_{3}^{2}}\right) / \xi \\
L_{3}=C_{3} / \xi \\
L_{4}=\sqrt{C_{4}} \\
L_{5}=\sqrt{C_{5}}
\end{array}\right.
$$

with $\xi=\sqrt{C_{1}+C_{2}+2 \sqrt{C_{1} C_{2}-C_{3}^{2}}}$.

\section{ADDITIONAL CONVERGENCE RESULTS}

\section{References}

[1] K. Acton and L. Graham-Brady. Meso-scale modeling of plasticity in composites. Computer Methods in Applied Mechanics and Engineering, 198:920-932, 2009. 

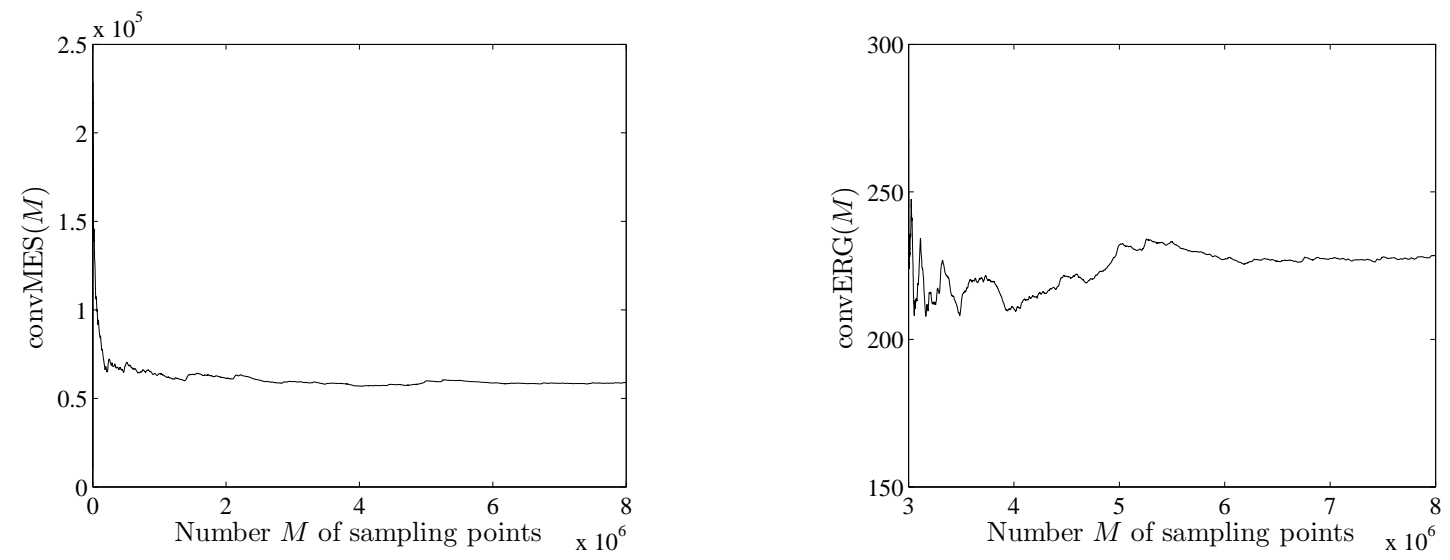

Figure 13: Graphs of mappings $M \mapsto \operatorname{convMES}(M)$ (left) and $M \mapsto \operatorname{convERG(} M)$ (right) for initial guess parametrized by $\lambda_{0}^{(2)}=-6.5$.

[2] V. Arsigny, P. Fillard, X. Pennec, and N. Ayache. Log-Euclidean metrics for fast and simple calculus on diffusion tensors. Magnetic Resonance in Medicine, 56:411-421, 2006.

[3] A. Bóna, I. Bucataru, and M.A. Slawinski. Coordinate-free characterization of the symmetry classes of elasticity tensors. Journal of Elasticity, 87:109-132, 2007.

[4] J.T. Browaeys and S. Chevrot. Decomposition of the elastic tensor and geophysical applications. Geophys. J. Int., 159:667-678, 2004.

[5] I. Bucataru and M. A. Slawinski. Invariant properties for finding distance in space of elasticity tensors. Journal of Elasticity, 94:97-114, 2009.

[6] P. Chadwick, M. Vianello, and S.C. Cowin. A new proof that the number of linear elastic symmetries is eight. Journal of the Mechanics and Physics of Solids, 49:2471-2492, 2001.

[7] T. M. Cover and J. A. Thomas. Elements of Information Theory. John Wiley \& Sons, New-York, 2006.

[8] S. Geman and Geman D. Stochastic relaxation, Gibbs distributions, and the Bayesian restoration of images. IEEE transactions on pattern analysis and machine intelligence, 6(6):721-741, 1984.

[9] R. Ghanem and P. Spanos. Stochastic finite elements: a Spectral Approach. Springer, New-York, 1991.

[10] S. Greene, Y. Liu, W. Chen, and W. K. Liu. Computational uncertainty analysis in multiresolution materials via stochastic constitutive theory. Computer Methods in Applied Mechanics and Engineering, 200:309-325, 2011.

[11] J. Guilleminot, A. Noshadravan, C. Soize, and R. G. Ghanem. A probabilistic model for bounded elasticity tensor random fields with application to polycrystalline microstructures. Computer Methods in Applied Mechanics and Engineering, 200:1637-1648, 2011. 

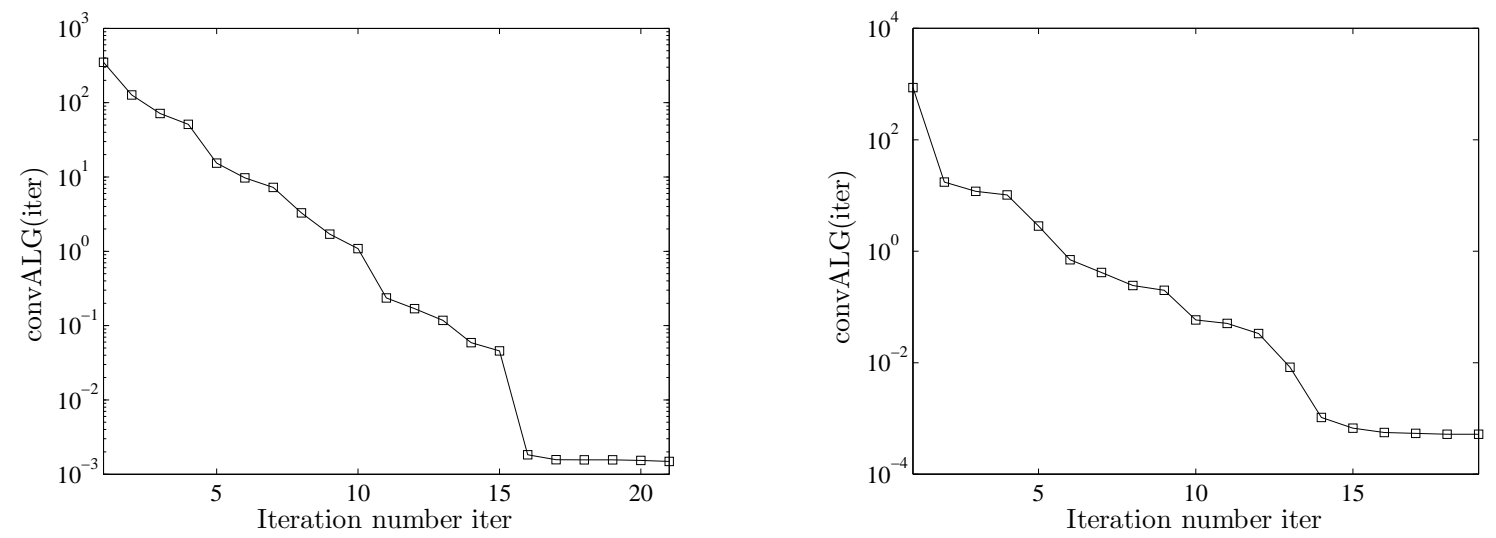

Figure 14: Graphs of iter $\mapsto$ convALG(iter) for medium (left) and larger (right) fluctuations.

[12] J. Guilleminot and C. Soize. A stochastic model for elasticity tensors with uncertain material symmetries. International Journal of Solids and Structures, 47:3121-3130, 2010.

[13] J. Guilleminot and C. Soize. Non-Gaussian positive-definite matrix-valued random fields with constrained eigenvalues: Application to random elasticity tensors with uncertain material symmetries. International Journal of Numerical Methods in Engineering, 86, 2011. DOI: 10.1002/nme.3212.

[14] J. Guilleminot and C. Soize. Stochastic modeling of anisotropy in multiscale analysis of heterogeneous materials: a comprehensive overview on random matrix approaches. Mechanics of Materials, 2011. Accepted for publication (Special Issue on "Evolving microstructures and anisotropies in engineering materials and biological tissues").

[15] W. K. Hastings. Monte Carlo sampling methods using Markov Chains and their applications. Biometrika, 57:97-109, 1970.

[16] R. A. Horn and C. R. Johnson. Matrix analysis. Cambridge University Press, New-York, USA, 1985.

[17] C. Huet. Application of variational concepts to size effects in elastic heterogeneous bodies. Journal of the Mechanics and Physics of Solids, 38(6):813-841, 1990.

[18] E. T. Jaynes. Information theory and statistical mechanics. Physical Review, 106/108(4/2):620630/171-190, 1957.

[19] J. N. Kapur and H. K. Kesavan. Entropy Optimization Principles with Applications. Academic Press, San Diego, 1992.

[20] P. E. Kloeden and E. Platen. Numerical Solution of Stochastic Differentials Equations. Springer, Berlin, 1992.

[21] M. Kochetov and M.A. Slawinski. Estimating effective elasticity tensors from christoffel equations. Geophysics, 74(5):WB67-WB73, 2009. 
[22] M. Kochetov and M.A. Slawinski. On obtaining effective orthotropic elasticity tensors. 62(2):149-166, 2009.

[23] M. Kochetov and M.A. Slawinski. On obtaining effective transversely isotropic elasticity tensors. Journal of Elasticity, 94(1):1-13, 2009.

[24] I. A. Kunin. An algebra of tensor operators and its applications to elasticity. Int. J. Engng Sci., 19:1551-1561, 1981.

[25] M.M. Mehrabadi and S.C. Cowin. Eigentensors of linear anisotropic elastic materials. 43(1):15-41, 1990.

[26] N. Metropolis, A. W. Rosenbluth, M. N. Rosenbluth, A. H. Teller, and E. Teller. Equation of State Calculations by Fast Computing Machines. Journal of Chemical Physics, 21(6):1087-1091, 1953.

[27] N. Metropolis and S. Ulam. The monte carlo method. J. Amer. Statist. Assoc., 44:335-341, 1949.

[28] M. Moakher. On the averaging of symmetric positivedefinite tensors. Journal of Elasticity, 82:273296, 2006.

[29] M. Moakher and A. N. Norris. The closest elastic tensor of arbitrary symmetry to an elasticity tensor of lower symmetry. Journal of Elasticity, 85:215-263, 2006.

[30] R. M. Neal. Slice sampling. Annals of statistics, 31(3):705-767, 2003.

[31] M. Ostoja-Starzewski. Random field models of heterogeneous materials. International Journal of Solids and Structures, 35(19):2429-2455, 1998.

[32] M. Ostoja-Starzewski. Microstructural Randomness and Scaling in Mechanics of Materials. Chapman and Hall-CRC, 2008.

[33] C. P. Robert and G. Casella. Monte Carlo Statistical Methods. Springer, New York, 2005.

[34] K. Sab. On the homogenization and the simulation of random materials. European Journal of Mechanics A/Solids, 11(5):585-607, 1992.

[35] J. Serra. Image analysis and Mathematical Morphology. Academic Press, London, 1982.

[36] C. E. Shannon. A mathematical theory of communication. Bell System Technical Journal, 27:379423/623-659, 1948.

[37] C. Soize. A nonparametric model of random uncertainties on reduced matrix model in structural dynamics. Probabilistic Engineering Mechanics, 15(3):277-294, 2000.

[38] C. Soize. Maximum entropy approach for modeling random uncertainties in transient elastodynamics. Journal of the Acoutical Society of America, 109(5):1979-1996, 2001.

[39] C. Soize. Random matrix theory for modeling uncertainties in computational mechanics. Computer Methods in Applied Mechanics and Engineering, 194:1333-1366, 2005.

[40] C. Soize. Non-gaussian positive-definite matrix-valued random fields for elliptic stochastic partial differential operators. Computer Methods in Applied Mechanics and Engineering, 195:26-64, 2006. 
[41] C. Soize. Construction of probability distributions in high dimension using the maximum entropy principle: Applications to stochastic processes, random fields and random matrices. International Journal of Numerical Methods in Engineering, 76:1583-1611, 2008.

[42] C. Soize. Generalized probabilistic approach of uncertainties in computational dynamics using random matrices and polynomial chaos decompositions. International Journal of Numerical Methods in Engineering, 81(8):939-970, 2009.

[43] C. Soize. Identification of high-dimension polynomial chaos expansions with random coefficients for non-gaussian tensor-valued random fields using partial and limited experimental data. Computer Methods in Applied Mechanics and Engineering, 199(33-36):2150-2164, 2010.

[44] Q.A. Ta, D. Clouteau, and R. Cottereau. Modeling of random anisotropic elastic media and impact on wave propagation. European Journal of Computational Mechanics, 19(1-2-3):241-253, 2010.

[45] M. Tootkaboni and L. Graham-Brady. A multi-scale spectral stochastic method for homogenization of multi-phase periodic composites with random material properties. International Journal of Numerical Methods in Engineering, 83:59-90, 2010.

[46] S. Torquato. Random Heterogeneous Materials: Microstructure and Macroscopic Properties. Springer, New-York, 2002.

[47] L. J. Walpole. Elastic Behavior of Composite Materials: Theoretical Foundations. Advances in Applied Mechanics, 21:169-242, 1981.

[48] L. J. Walpole. Fourth-rank tensors of the thirty-two crystal classes: Multiplication tables. Proc. R. Soc. Lond. A, 391:149-179, 1984.

[49] X. F. Xu and L. Graham-Brady. A stochastic computational method for evaluation of global and local behavior of random elastic media. Computer Methods in Applied Mechanics and Engineering, 194:4362-4385, 2005.

[50] K. Yosida. Functional Analysis. Springer, Berlin, 1995. 